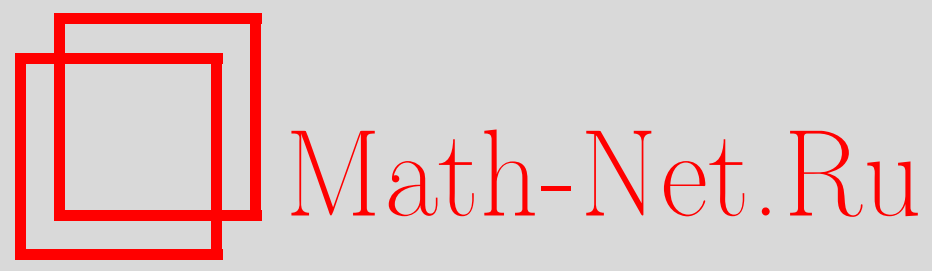

K. К. Щукин, В. В. Гушан, Лупы порядка шесть, Дискрет. матем., 2002, том 14, выпуск 2, 134-160

DOI: https://doi.org/10.4213/dm247

Использование Общероссийского математического портала Math-Net.Ru подразумевает, что вы прочитали и согласны с пользовательским соглашением http://www.mathnet.ru/rus/agreement

Параметры загрузки:

IP : 54.147 .182 .235

26 апреля 2023 г., 14:44:04

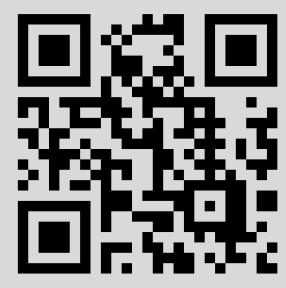


УдК 512.6

\title{
Лупы порядка шесть
}

\author{
(c) 2002 г. .К. Щукин, В. В. Гушан
}

Определены группы автоморфизмов, классы антиизоморфизма и образующие групп внутренних подстановок луп порядка шесть.

Авторы посвящают эту работу памяти В. Д. Белоусова (1925-1988).

\section{1. Введение}

Данная работа является алгебраическим продолжением статьи [6], которая прокомментирована Денешом и Кидвеллом (см. [4], гл. 4, §3) следующим образом: "Шёнхард показал, что существует 2 класса изотопии для латинских квадратов порядка 5 и 22 класса изотопии для латинских квадратов порядка 6. Он показал, что имеется 6 классов изоморфизма для приведенных $5 \times 5$ квадратов и 109 классов изоморфизма для приведенных $6 \times 6$ квадратов, а общее число приведенных квадратов порядков 5 и 6 равно, соответственно, 56 и 9408. Все эти результаты правильны." Наша независимая компьютерная проверка подтверждает эти данные.

Каждому приведенному латинскому $n \times n$ квадрату, определенному на множестве $Q=\{1,2, \ldots, n\}$ соответствует точно одна лупа $(Q, \cdot)$ с операцией умножения, определяемой таблицей с входными строкой и столбцом вида $1,2, \ldots, n$. Такая лупа называется канонической лупой или $c(n)$-лупой. Мы исследует только приведенные $n \times n$ квадраты для $c(n)$-луп при $n=5,6$. Наша нумерация классов изоморфизма $c(n)$-луп отличается от нумерации в [4]. Кроме того, для определения классов изоморфизма $c(n)$-луп использованы их левые представления в отличии от правых представлений в [1]. Алгоритм, используемый для вычислений, мы не выписываем. Он основан на доказанных ниже теоремах. Другие используемые нами факты о квазигруппах содержатся в монографии В. Д. Белоусова [2] и в [5].

Авторы благодарят Юрия Рогозина за полезные дискуссии и поддержку.

\section{2. Вычисления}

Для лупы $(Q, \cdot)$ и элемента $a \in Q$ определены две подстановки

$$
L_{a}: x \rightarrow a x \quad R_{a}: x \rightarrow x a,
$$

называемые левой и правой трансляцией соответственно. Положим

$$
\begin{aligned}
& Q_{L}=\left\{L_{a} \mid a \in Q\right\}, \\
& Q_{R}=\left\{R_{a} \mid a \in Q\right\} .
\end{aligned}
$$


Группа, порожденная этими двумя множествами, называется группой трансляций лупы $(Q, \cdot)$ и обозначается $M(Q, \cdot)$ или просто $M(Q)$. Группу всех автоморфизмов и множество всех антиавтоморфизмов лупы $(Q, \cdot)$ обозначим соответственно через Aut $Q$ и AAut $Q$.

Следующая теорема является естественным обобщением теорем 9 и 10 из [1].

Теорема 1. Пусть $\varphi-$ биекция лупы $(Q, \cdot)$ на лупу $(Q$, о) такая, что

$$
\varphi M(Q, \cdot) \varphi^{-1}=M(Q, \circ) .
$$

Тогда $\varphi$ - изоморфизм (антиизоморфизм) в том и только том случае, когда $\varphi$ отображает единицу $(Q, \cdot)$ в единииу $\left(Q\right.$, о) и $\varphi Q_{L} \varphi^{-1}=Q_{L^{\circ}}$ (соответственно, $\varphi Q_{L} \varphi^{-1}=Q_{R^{\circ}}$ ), где $Q_{L^{\circ}}$ и $Q_{R^{\circ}}$ - множества всех левых и правых транслячий на $(Q$, о).

В случае $(Q, \cdot)=(Q$, о) получаем теорему Алберта об автоморфизмах и антиавтоморфизмах $(Q, \cdot)$.

Пусть $(Q, \cdot)$ - лупа порядка $n$, определенная на множестве $Q=\{1,2, \ldots, n\}$.

Определение 1. Лупа $(Q, \cdot)$ называется канонической или $c(n)$-лупой, если ее таблица умножения может быть представлена в виде

\begin{tabular}{c|cccc}
$\cdot$ & 1 & 2 & $\ldots$ & $n$ \\
\hline 1 & 1 & 2 & $\ldots$ & $n$ \\
2 & 2 & $*$ & $\ldots$ & $*$ \\
$\cdot$ & $\cdot$ & $\cdot$ & $\ldots$ & $\cdot$ \\
$\cdot$ & $\cdot$ & $\cdot$ & $\ldots$ & $\cdot$ \\
$\cdot$ & $\cdot$ & $\cdot$ & $\ldots$ & $\cdot$ \\
$n$ & $n$ & $*$ & $\ldots$ & $*$
\end{tabular}

Это определение эквивалентно следующему определению.

Определение 2. Лупа $(Q, \cdot)$ называется канонической тогда и только тогда, когда

$$
L_{i}(1)=i=R_{i}(1), \quad i=1,2, \ldots, n .
$$

С использованием теоремы 1 легко доказывается следующее утверждение.

Теорема 2. Каждая лупа, определенная на множестве

$$
Q=\{1,2, \ldots, n\},
$$

изоморфна некоторой с(n)-лупе.

\section{1. Классификация классов изоморфизма по отношению к классам изотопии}

Все лупы порядка пять и шесть могут быть распределены в 24 класса изотопии. В каждом классе мы найдем число канонических луп и число классов изоморфизма. Лупы порядка пять можно разбить на два класса изотопии, обозначаемых ниже I и II. Первый класс содержит 50 канонических луп и 5 классов изоморфизма. Второй класс содержит 6 канонических луп и только один класс изоморфизма. Полученные результаты для луп порядка шесть представлены в табл. 1, в которой первая строка содержит названия классов изотопии, вторая - число канонических луп в этом классе, третья - число классов изоморфизма. 
Таблица 1. Лупы порядка шесть

\begin{tabular}{||c|c|c|c|c|c|c|c|c|c|c|c}
\hline \hline I & II & III & IV & V & VI & VII & VIII & IX & X & XI & XII \\
\hline 60 & 180 & 120 & 1080 & 20 & 540 & 360 & 36 & 360 & 1080 & 540 & 360 \\
\hline 1 & 2 & 3 & 10 & 1 & 5 & 4 & 2 & 4 & 12 & 7 & 4 \\
\hline \hline
\end{tabular}

\begin{tabular}{c|c|c|c|c|c|c|c|c|c||}
\hline \hline XIII & XIV & XV & XVI & XVII & XVIII & XIX & XX & XXI & XXII \\
\hline 36 & 540 & 1080 & 1080 & 120 & 1080 & 120 & 540 & 36 & 40 \\
\hline 2 & 7 & 10 & 9 & 3 & 10 & 3 & 7 & 2 & 1 \\
\hline \hline
\end{tabular}

Заметим, что все лупы порядка пять из класса I и все лупы порядка шесть из классов $\mathrm{I}$ и $\mathrm{V}$ изоморфны циклическим группам $Z_{5}, Z_{6}$ и симметрической группе подстановок $S_{3}$, соответственно. Класс XXII представлен некоммутативной и неассоциативной лупой, имеющей номер 38 в списке Алберта (см. [1]) классов луп порядка шесть с подлупами порядка три.

Возникает вопрос: не является ли класс XXII частью некоторого неизвестного класса изотопии луп, совпадающего с его классом изоморфизма?

\section{2. Автоморфизмы групп}

Ниже все группы автоморфизмов луп задаются левыми представлениями и используются следующие обозначения:

$Z_{n}$ - циклическая группа порядка $n$,

$K_{4} \cong Z_{2} \times Z_{2}-$ группа Клейна,

$S_{n}$ - симметрическая группа подстановок степени $n$,

$A_{n}$ - знакопеременная группа порядка $n$,

$Z_{5} \lambda Z_{4} \cong\left\langle x, y \mid x^{5}=1, x^{4}=1=x^{3}\right\rangle-$ полупрямое произведение $Z_{5}$ и $Z_{4}$. Это метациклическая группа порядка 20. Все ее собственные подгруппы являются циклическим.

Символы $\mathrm{I}_{k}, \mathrm{II}_{k}, \ldots, k=1,2, \ldots$, используются всюду ниже для перечисления классов изоморфизма в классах изотопии I, II, ...

Замечание 1. Индикатором коммутативности лупы в наших вычислениях является равенство $\mathrm{AAut} Q=$ Aut $Q$. Так, из каталогов мы имеем единственный коммутативный класс II (циклические группы) порядка пять и следующие группы порядка шесть: I (циклические группы), $\mathrm{II}_{1}, \mathrm{XIII}_{1}, \mathrm{XIV}_{1}, \mathrm{XIV}_{2}, \mathrm{XV}_{1}$ (единственный с $\{\varepsilon\}=$ AAut $Q=$ Aut $Q$ ), $\mathrm{XV}_{3}$ и $\mathrm{XIX}_{1}$. 
Таблица 2.

\begin{tabular}{|c|c|c|c|c|}
\hline $\begin{array}{l}\text { Типы } \\
\text { групп }\end{array}$ & $\begin{array}{c}\text { порядок } \\
\text { пять }\end{array}$ & $\begin{array}{c}\text { число } \\
\text { классов }\end{array}$ & $\begin{array}{c}\text { порядок } \\
\text { шесть }\end{array}$ & $\begin{array}{c}\text { число } \\
\text { классов }\end{array}$ \\
\hline $\begin{array}{l}\text { единичная } \\
\text { группа }\{\varepsilon\}\end{array}$ & $I_{5}$ & 1 & $\begin{array}{c}\mathrm{II}_{2} \mathrm{IV}_{1,3,5-10} \mathrm{VI}_{1-4} \mathrm{VII}_{2,4} \mathrm{IX}_{2,3} \\
\mathrm{X}_{2,3,7-12} \mathrm{XI}_{5,6,7} \mathrm{XII}_{2,3} \mathrm{XIV}_{3,6,7} \\
\mathrm{XV}_{1,2,4,6-10} \mathrm{XVI}_{1-9} \mathrm{XVII}_{1,2,5-10} \mathrm{XX}_{2,6,7}\end{array}$ & 61 \\
\hline$Z_{2}$ & & & $\begin{array}{c}\mathrm{I}_{1} \mathrm{II}_{1} \mathrm{III}_{2} \mathrm{IV}_{2,4} \mathrm{VI}_{5} \mathrm{VII}_{1,3} \mathrm{IX}_{1,4} \\
\mathrm{XI}_{1,3} \mathrm{XII}_{1,4} \mathrm{XIV}_{1,4} \mathrm{XV}_{3,5} \mathrm{XVII}_{2} \\
\mathrm{XVIII}_{3,4} \mathrm{XIX}_{1} \mathrm{XX}_{1,4}\end{array}$ & 24 \\
\hline$Z_{3}$ & $\mathrm{I}_{1,2,4}$ & 3 & $\mathrm{III}_{3} \mathrm{XVII}_{3} \mathrm{XIX}_{3} \mathrm{XXII}_{1}$ & 4 \\
\hline$Z_{4}$ & $\mathrm{II}_{1}$ & & $\begin{array}{c}\mathrm{VIII}_{2} \mathrm{XI}_{2,4} \mathrm{XIII}_{2} \mathrm{XIV}_{2,5} \\
\mathrm{XX}_{3,5} \mathrm{XXI}_{2}\end{array}$ & 9 \\
\hline$K_{4}$ & & & $\mathrm{X}_{1,4,5,6}$ & 4 \\
\hline$S_{3}$ & & & $\mathrm{III}_{1} \mathrm{~V}_{1} \mathrm{XVII}_{1} \mathrm{XIX}_{2}$ & 4 \\
\hline$A_{4}$ & $\mathrm{I}_{3}$ & 1 & & \\
\hline$Z_{5} \lambda Z_{4}$ & & & $\mathrm{VIII}_{1} \mathrm{XIII}_{1} \mathrm{XXI}_{1}$ & 3 \\
\hline
\end{tabular}

\section{3. Группы внутренних подстановок}

Пусть $(Q, \cdot)$ - лупа и $e-$ ее единица. Группа $I(Q)=I(Q, \cdot)$ внутренних подстановок лупы $(Q, \cdot)$ определяется следующим образом:

$$
I(Q)=\{\varphi \in S(Q) \mid \varphi(e)=e\}
$$

где $S(Q)$ - симметрическая группа подстановок множества $Q$. Группа $I(Q)$ порождается подстановками

$$
S_{a, b}=L_{a b}^{-1} L_{a} L_{b}, \quad T_{a, b}=R_{a b}^{-1} R_{b} R_{a}, \quad Z_{a}=L_{a}^{-1} R_{a}
$$

Используя эти факты, мы создали алгоритм вычисления множества $\langle I(Q)\rangle$ всех таких порождающих подстановок. В большинстве случаев $\operatorname{Card}\langle I(Q)\rangle$ - довольно большое число (иногда большее 50, см. $X_{1}$ и т. д). Довольно обычными являются ситуациии, когда $\varphi, \psi, \theta \in\langle I(Q)\rangle, \psi=\varphi^{2}, \theta=\varphi^{-1}$ и т. п. Заметим, что множества $\langle I(Q)\rangle$ несут определенную информацию о строении групп $I(Q)$ и $M(Q)$. Кроме классов изоморфизма групп существует также одна лупа порядка шесть для которой $I(Q)$ легко определяется. Так, $I(Q)$ изоморфна $K_{4}$ в $\mathrm{II}_{1,2}$ и $S_{3}$ в классах $\mathrm{XIX}_{2}$ и XXII. В случае $\mathrm{XIX}_{2}$ справедливо также соотношение $I(Q)=$ Aut $Q \cong S_{3}$, то есть все лупы класса $\mathrm{XIX}_{2}$ являются $A$-лупами (лупа называется $A$-лупой, если только $I(Q) \subseteq$ Aut $Q$ ). Вообще, $\mathrm{XIX}_{2}$ - единственный класс изоморфизма $A$-луп среди луп порядка пять и шесть. Дадим следующее определение.

Определение 3. Лупа $(Q, \cdot)$ называется полной, если ее центр есть $e$ и $I(Q)=$ Aut $Q$.

В связи с описанной выше ситуацией возникает вопрос: существуют ли неассоциативные полные лупы $(Q, \cdot)$ порядка $n \geqslant 6$ с условием $I(Q)=$ Aut $Q$ ? 
Таблица 3.

\begin{tabular}{|c|c|c|c|c|c|c|}
\hline № в списке Алберта & 17 & 18 & 19 & 20 & 21 & \\
\hline Класс изоморфизма & $\mathrm{I}_{2}$ & $\mathrm{I}_{5}$ & $\mathrm{I}_{4}$ & $\mathrm{I}_{3}$ & $\mathrm{I}_{1}$ & II \\
\hline$\varphi \varphi L_{a} \varphi^{-1}=L_{\varphi(a)}^{\circ}$ & (24) & $\varepsilon$ & (23) & (23) & $\varepsilon$ & $\varepsilon$ \\
\hline$M(Q) \cong$ & \multicolumn{5}{|c|}{ Симметрическая группа $S_{5}$} & $\overline{Z_{5}}$ \\
\hline$I(Q) \cong$ & \multicolumn{5}{|c|}{ Симметрическая группа $S_{4}$} & $\{\varepsilon\}$ \\
\hline Aut $Q \cong$ & $Z_{3}$ & $\{\varepsilon\}$ & $Z_{3}$ & $A_{4}$ & $Z_{3}$ & $Z_{4}$ \\
\hline Card(AAut $Q$ ) & 0 & 1 & 3 & 12 & 0 & 0 \\
\hline $\operatorname{Card}\left\{a \in Q \mid a^{2}=1\right\}$ & 1 & 0 & 1 & 4 & 0 & 0 \\
\hline
\end{tabular}

\section{4. Группы, ассоциированные с лупами}

Мы не пытались определять группы, ассоциированные со всеми $c(6)$-лупами, представляющими классы изоморфизма. Иногда они легко определяются их образующими элементами. Каждый случай требует специального анализа и своей техники (см. ниже).

\section{5. Список Алберта $c(n)$-луп}

Все шесть классов изоморфизма луп порядка пять описаны Албертом в [1]. Для $Q=$ $\{1,2,3,4,5\}$ группа $M(Q)$ примитивна. Все их нетрудно определить также по табл. 1 из [7]. Для $Q \cong Z_{5}$ все включения $M(Q) \supset I(Q) \supset$ Aut $Q$ собственные. Все лупы порядка пять просты. Для луп из II и $\mathrm{I}_{5}$ группы $M(Q)$ не имеют собственных подгрупп. Кроме того, в [1] отмечено, что классы $\mathrm{I}_{1}$ и $\mathrm{I}_{2}$ антиизоморфны.

Список представлений всех двенадщати классов изоморфизма луп порядка шесть с подлупами порядка три дан в [1]. Используя этот список, мы делим все неассоциативные $c(6)$-лупы, соответствующие этим лупам, на две части. Первая часть содержит $c(6)$-лупы с номерами $29, \ldots, 35$, вторая - $c(6)$-лупы с номерами $36,37,38$. Будем говорить, что лупы из первой части имеют тип один, из второй - тип два. Все эти лупы имеют нормальную подлупу $H=\{1,2,3\}$. Стабилизатор подлупы $H$ в $M(Q)$, то есть

$$
\text { st } H=\{\varphi \in(M) Q \mid \varphi(H)=H\}
$$

является нормальным делителем $M(Q)$, так что $M(Q / H) \cong M(Q) /$ st $H$. Таким образом, Card $H=($ st $H: I(Q))$ и, следовательно, по теореме Лагранжа

$$
\operatorname{Card} M(Q)=(\text { st } H: I(Q))(M(Q): \text { st } H) \text {. }
$$

Мы применим эти факты к вычислению $M(Q)$.

Рассмотрим тип один. Пусть $(Q, \cdot)-c(6)-$ лупа с номером 29. Она коммутативна и, как легко проверить, $I(Q) \cong Z_{2} \times S_{3} \cong D_{6}-$ группа диэдра. Таким образом Card st $H=$ 3 Card $I(Q)=36$ и Card $M(Q)=2$ Card st $H=72$. Но $L_{3} L_{4}=(1,6)(2,4)(3,5) \notin$ st $H$, $(1,2)(4,5) \in$ st $H$ и $(1,2)(4,5) L_{3} L_{4}=L_{3} L_{4}(4,6)$. Следовательно, $M(Q)=$ st $H \lambda\left\langle L_{3} L_{4}\right\rangle$. Очевидно, что st $H$, и следовательно, $M(Q)$ могут быть найдены в явном виде. Все лупы типа один имеют одну и ту же ассоциированную группу.

Рассмотрим тип два. Пусть $(Q, \cdot)$ - лупа с номером 38 . Это $c(6)$-лупа $(Q$, , $)$, которая представляет класс XXII. Нетрудно видеть, что $I(Q) \cong S_{3}$. Подобно предыдущему случаю 
Таблица 4.

\begin{tabular}{||c|c|c|c|c|c|}
\hline \hline № в списке Алберта & & & 30 & 31 & \\
\hline Класс изоморфизма & $\mathrm{I}$ & $\mathrm{V}$ & $\mathrm{III}_{2}$ & $\mathrm{XVII}_{2}$ & \\
\hline$\varphi: \varphi L_{a} \varphi^{-1}=L_{\varphi(a)}^{\circ}$ & & & \multicolumn{2}{|c|}{$(25)(34)$} & $(25)$ \\
\hline$M(Q) \cong$ & $Z_{6}$ & $S_{3} \times S_{9}$ & \multicolumn{2}{|c|}{ Группа типа один } \\
\hline$I(Q) \cong$ & $\{\varepsilon\}$ & $S_{3}$ & \multicolumn{2}{|c|}{ Группа диэдра $D_{6}$} \\
\hline Aut $Q \cong$ & $Z_{2}$ & $S_{3}$ & $Z_{2}$ & $Z_{2}$ \\
\hline Card(AAut $G)$ & 0 & 6 & 0 & 0 \\
\hline \hline
\end{tabular}

\begin{tabular}{c|c|c|c|c|c|c|c||}
\hline \hline 32 & 33 & 34 & 35 & 29 & 36 & 37 & 38 \\
\hline $\mathrm{XVII}_{1}$ & $\mathrm{XVII}_{3}$ & $\mathrm{III}_{1}$ & $\mathrm{III}_{3}$ & $\mathrm{XIX}_{1}$ & $\mathrm{XIX}_{2}$ & $\mathrm{XIX}_{3}$ & $\mathrm{XXII}$ \\
\hline$\varepsilon$ & $\varepsilon$ & $(25)$ & $\varepsilon$ & $(2564)$ & $(24)(36)$ & $(56)$ & $(56)$ \\
\hline \multicolumn{3}{c||}{ Группа типа один } & \multicolumn{5}{|c}{ Группа типа два } \\
\hline \multicolumn{3}{|c}{ Группа диэдра $D_{6}$} & $S_{3}$ & $S_{3}$ & $S_{3}$ & $S_{3}$ \\
\hline$Z_{3}$ & $Z_{3}$ & $S_{3}$ & $Z_{3}$ & $Z_{2}$ & $S_{3}$ & $Z_{3}$ & $Z_{3}$ \\
\hline 0 & 0 & 0 & 0 & 0 & 6 & 3 & 3 \\
\hline \hline
\end{tabular}

мы докажем, что $M(Q)=$ st $H \lambda\left\langle L_{4}^{3}\right\rangle$, где $L_{4}^{3}=(1,6)(2,4)(3,5)$. Для всех луп типа два Card st $H=18$ и Card $M(Q)=36$. Кроме того, st $H$ изоморфна группе

$$
\left\langle x, y, z \mid x^{2}=y^{2}=z^{2}=(x y)^{3}=(x z)^{3}=(x y z)^{2}=1\right\rangle
$$

из табл. 1 в [3]. Этот изоморфизм задается отображением

$$
x \rightarrow(1,2)(4,5), \quad y \rightarrow(2,3)(4,6), \quad z \rightarrow(2,3)(5,6) .
$$

Полученные результаты представлены в табл. 4.

Рассмотрим ряд контрпримеров. По табл. 4 лупы с номерами 29, 36 и 37 из списка Алберта содержатся в классах изоморфизма $\mathrm{XIX}_{1}, \mathrm{XIX}_{2}$ и $\mathrm{XIX}_{3}$ соответственно. Эти три класса составляют класс изотопии XIX. Следовательно, утверждение Алберта ([1], стр. 419), что “каждая лупа, изотопная лупе 36, изоморфна лупам 36, или 37" неточно. Должно быть: “каждая лупа, изотопная лупе 36, изоморфна одной из луп 29, 36, 37”. По той же самой причине формулировка другого утверждения Алберта (на той же странице в [1]) “лупа изотопна коммутативной лупе $(Q, \cdot)$ порядка шесть с подлупой порядка три, тогда и только тогда, когда она изоморфна $(Q, \cdot)$ ” является, очевидно, неправильной.

\section{3. Классификационные каталоги для луп порядка пять и шесть}

В этой части мы представляем таблицы, содержащие наши результаты. Первый столбец каждой таблицы содержит номер соответствующего класса изоморфизма, второй - номера канонических луп (CL) в этом классе. Строка с $Q_{L}$ содержит все левые представления канонических луп этого класса. Автоморфизмы канонической лупы $(Q, \cdot)$ перечислены в строке с Aut $Q$, антиавтоморфизмы - в строке с AAut $Q$. Строка с $\langle I(Q)\rangle$ содержит все образующие элементы группы $I(Q)$. 


\section{Порядок пять}

\begin{tabular}{|c|c|c|c|}
\hline № & $\mathrm{CL}$ & & Класс изотопии I \\
\hline \multirow{4}{*}{1} & \multirow{4}{*}{8} & $Q_{L}$ & $\varepsilon,(12)(345),(13524),(14325),(15423)$ \\
\hline & & Aut $Q$ & $\{\varepsilon,(345),(354)\} \cong Z_{3}$ \\
\hline & & AAut $Q$ & $\varnothing$ \\
\hline & & $\langle I(Q)\rangle$ & $\begin{array}{l}(45),(34),(345),(354),(35),(23)(45),(234),(2345),(2354), \\
(2453),(245),(24)(35),(2435),(2543),(253),(2534),(25)(34)\end{array}$ \\
\hline \multirow{4}{*}{2} & \multirow{4}{*}{8} & $Q_{L}$ & $\varepsilon,(12)(345),(135)(24),(143)(25),(154)(23)$ \\
\hline & & Aut $Q$ & $\{\varepsilon,(345),(354)\} \cong Z_{3}$ \\
\hline & & AAut $Q$ & $\varnothing$ \\
\hline & & $\langle I(Q)\rangle$ & $\begin{array}{l}(45),(34),(345),(354),(35),(23)(45),(234),(2345),(2354), \\
(2453),(245),(24)(35),(2435),(2543),(253),(2534),(25)(34)\end{array}$ \\
\hline \multirow{4}{*}{3} & \multirow{4}{*}{2} & $Q_{L}$ & $\varepsilon,(12)(345),(13)(254),(14)(235),(15)(243)$ \\
\hline & & Aut $Q$ & $\begin{array}{l}\{\varepsilon,(345),(354),(23)(45),(234),(235),(243),(245),(24)(35),(253), \\
(254),(25)(34)\} \cong A_{4}\end{array}$ \\
\hline & & AAut $Q$ & $\begin{array}{l}(45),(34),(35),(23),(2345),(2354),(2453),(24),(2435),(2543), \\
(25),(2534)\end{array}$ \\
\hline & & $\langle I(Q)\rangle$ & $\begin{array}{l}(45),(34),(345),(354),(35),(23),(234),(235),(243),(24),(245), \\
(253),(254),(25)\end{array}$ \\
\hline \multirow{4}{*}{4} & \multirow{4}{*}{8} & $Q_{L}$ & $\varepsilon,(12)(345),(13254),(14235),(15243)$ \\
\hline & & Aut $Q$ & $\{\varepsilon,(345),(354)\} \cong Z_{3}$ \\
\hline & & AAut $Q$ & $(45),(34),(35)$ \\
\hline & & $\langle I(Q)\rangle$ & $\begin{array}{l}\text { (45), (34), (345), (354), (35), (23), (234), (2345), (2354), (235), } \\
(243),(2453),(24),(245),(2435),(2543),(253),(254),(25),(2534)\end{array}$ \\
\hline \multirow{4}{*}{5} & \multirow{4}{*}{24} & $Q_{L}$ & $\varepsilon,(123)(45),(13524),(14325),(15342)$ \\
\hline & & Aut $Q$ & $\{\varepsilon\}$ \\
\hline & & AAut $Q$ & (24) \\
\hline & & $\langle I(Q)\rangle$ & $\begin{array}{l}(45),(34),(345),(354),(35),(23),(234),(2354),(235),(243), \\
(2453),(24),(245),(24)(35),(2435),(253),(254),(25),(2534)\end{array}$ \\
\hline
\end{tabular}

\begin{tabular}{||l|l|l|l||}
\hline \hline № & CL & \multicolumn{2}{|c||}{ Класс изотопии II } \\
\hline \multirow{3}{*}{1} & \multirow{3}{*}{6} & $Q_{L}$ & $\varepsilon,(12345),(13524),(14253),(15432)$ \\
\cline { 3 - 4 } & & Aut $Q$ & $\{\varepsilon,(2354),(2453),(25)(34)\} \cong Z_{4}$ \\
\cline { 2 - 3 } & & AAut $Q$ & $=$ Aut $Q$ \\
\cline { 3 - 4 } & & $I(Q)$ & $\{\varepsilon\}$ \\
\hline
\end{tabular}

\section{Порядок шесть}

\begin{tabular}{||l|l|l|l||}
\hline \hline № & CL & \multicolumn{2}{|c||}{ Класс изотопии I } \\
\hline \multirow{3}{*}{1} & \multirow{3}{*}{60} & $Q_{L}$ & $\varepsilon,(12)(34)(56),(135)(246),(145236),(153)(264),(163254)$ \\
\cline { 3 - 4 } & & Aut $Q$ & $\{\varepsilon,(35)(46)\} \cong Z_{2}$ \\
\cline { 2 - 3 } & & AAut $Q$ & $=$ Aut $Q$ \\
\cline { 2 - 3 } & & $I(Q)$ & $\{\varepsilon\}$ \\
\hline
\end{tabular}




\begin{tabular}{||c|c|c|l||}
\hline \hline № & CL & \multicolumn{2}{|c||}{ Класс изотопии II } \\
\hline \multirow{3}{*}{1} & \multirow{3}{*}{60} & $Q_{L}$ & $\varepsilon,(12)(34)(56),(135)(246),(145236),(154263),(164)(253)$ \\
\cline { 3 - 4 } & & Aut $Q$ & $\{\varepsilon,(36)(45)\} \cong Z_{2}$ \\
\cline { 3 - 4 } & & AAut $Q$ & $=$ Aut $Q$ \\
\cline { 3 - 4 } & $I(Q)$ & $\{(56),(34)\} \cong K_{4}$ \\
\hline \multirow{3}{*}{2} & \multirow{3}{*}{120} & $Q_{L}$ & $\varepsilon,(12)(34)(56),(135)(246),(145236),(153264),(163)(254)$ \\
\cline { 3 - 4 } & & Aut $Q$ & $\{\varepsilon\}$ \\
\cline { 3 - 4 } & & AAut $Q$ & $(35)(46)$ \\
\cline { 3 - 4 } & & $I(Q)$ & $\{\varepsilon,(56),(34),(34)(56)\} \cong K_{4}$ \\
\hline
\end{tabular}

\begin{tabular}{|c|c|c|c|}
\hline № & $\overline{\mathrm{CL}}$ & & Класс изотопии III \\
\hline \multirow{4}{*}{1} & \multirow{4}{*}{20} & $\overline{Q_{L}}$ & $\overline{\varepsilon,(12)(34)(56),(135)(246),(14)(25)(36),(153)(264),(16)(23)(45)}$ \\
\hline & & Aut $Q$ & $\{\varepsilon,(35)(46),(246),(24)(35),(264),(26)(35)\} \cong S_{3}$ \\
\hline & & AAut $Q$ & $\varnothing$ \\
\hline & & $\langle I(Q)\rangle$ & $(46),(35)(46),(24),(246),(24)(35),(264),(26),(26)(35)$ \\
\hline \multirow{4}{*}{2} & \multirow{4}{*}{60} & $Q_{L}$ & $\varepsilon,(12)(34)(56),(135246),(145)(263),(154)(236),(164253)$ \\
\hline & & Aut $Q$ & $\{\varepsilon,(36)(45)\} \cong Z_{2}$ \\
\hline & & AAut $Q$ & $\varnothing$ \\
\hline & & $\langle I(Q)\rangle$ & $(45),(36)(45),(23)(45),(236),(263),(26)(45)$ \\
\hline \multirow{4}{*}{3} & \multirow{4}{*}{40} & $Q_{L}$ & $\varepsilon,(123)(456),(132)(465),(142536),(152634),(162435)$ \\
\hline & & Aut $Q$ & $\{\varepsilon,(456),(465)\} \cong Z_{3}$ \\
\hline & & AAut $Q$ & $\varnothing$ \\
\hline & & $\langle I(Q)\rangle$ & $(56),(45),(456),(465),(46),(23)(56),(23)(45),(23)(46)$ \\
\hline
\end{tabular}

\begin{tabular}{|c|c|c|c|}
\hline № & $\mathrm{CL}$ & & Класс изотопии IV \\
\hline \multirow[b]{4}{*}{1} & \multirow[b]{4}{*}{120} & $\overline{Q_{L}}$ & $\varepsilon,(12)(34)(56),(135)(246),(142536),(154)(263),(164523)$ \\
\hline & & Aut $Q$ & $\{\varepsilon\}$ \\
\hline & & AAut $Q$ & $\varnothing$ \\
\hline & & $\langle I(Q)\rangle$ & $\begin{array}{l}(56),(46),(34),(34)(56),(3456),(346),(35)(46),(3654),(364), \\
(36),(234),(234)(56),(2345),(23456),(235),(23546),(2463), \\
(245),(2465),(2435),(245)(36),(25643),(2563),(254),(2564), \\
(256),(25)(46),(256)(34),(25)(346),(25346),(2654),(264),(26), \\
(26534),(26)(34),(26)(35)\end{array}$ \\
\hline \multirow[b]{4}{*}{2} & \multirow[b]{4}{*}{60} & $Q_{L}$ & $\varepsilon,(12)(34)(56),(135)(246),(145263),(154236),(164)(253)$ \\
\hline & & Aut $Q$ & $\{\varepsilon,(36)(45)\} \cong Z_{2}$ \\
\hline & & AAut $Q$ & $\varnothing$ \\
\hline & & $\langle I(Q)\rangle$ & $\begin{array}{l}(56),(45),(456),(465),(46),(34),(345),(3456),(354),(35), \\
(3654),(36)(45),(23)(45),(23)(465),(2346),(2356),(2365),(236), \\
(2456),(246),(2436),(24536),(2543),(253),(2563),(25463), \\
(2643),(2653),(263),(26)(45),(2634),(26)(345)\end{array}$ \\
\hline
\end{tabular}




\begin{tabular}{|c|c|c|c|}
\hline № & $\mathrm{CL}$ & & Класс изотопии IV \\
\hline \multirow[b]{4}{*}{3} & \multirow[b]{4}{*}{120} & $Q_{L}$ & $\varepsilon,(12)(34)(56),(135246),(14)(2536),(154263),(1645)(23)$ \\
\hline & & Aut $Q$ & $\{\varepsilon\}$ \\
\hline & & AAut $Q$ & $\varnothing$ \\
\hline & & $\langle I(Q)\rangle$ & $\begin{array}{l}(56),(45),(456),(465),(46),(34),(34)(56),(3456),(346), \\
(35)(46),(3654),(23)(56),(234)(56),(23465),(2354),(235), \\
(245),(2435),(24635),(2436),(245)(36),(2543),(25643),(254), \\
(256),(25)(46),(2534),(256)(34),(2536),(26543),(2654),(264), \\
(26534),(26)(354),(26)(35)\end{array}$ \\
\hline \multirow[b]{4}{*}{4} & \multirow[b]{4}{*}{60} & $Q_{L}$ & $\varepsilon,(12)(34)(56),(135246),(1425)(36),(153264),(1623)(45)$ \\
\hline & & Aut $Q$ & $\{\varepsilon,(35)(46)\} \cong Z_{2}$ \\
\hline & & AAut $Q$ & $\varnothing$ \\
\hline & & $\langle I(Q)\rangle$ & $\begin{array}{l}(56),(45),(456),(465),(46),(34),(3465),(346),(3564),(35)(46), \\
(364),(36),(234),(2346),(23564),(2356),(2463),(24),(246), \\
(24)(35),(24)(365),(2436),(2564),(256),(2534),(25346),(2654), \\
(264),(26),(2645),(26)(354),(26)(35)\end{array}$ \\
\hline \multirow{5}{*}{5} & \multirow[b]{4}{*}{20} & $Q_{L}$ & $\varepsilon,(12)(3456),(135246),(143265),(154)(236),(164253)$ \\
\hline & & Aut $Q$ & $\{\varepsilon\}$ \\
\hline & & AAut $Q$ & $\{\varnothing\}$ \\
\hline & & $\langle I(Q)\rangle$ & $\begin{array}{l}(45),(456),(46),(3456),(346),(35),(35)(46),(36)(45),(23)(45), \\
(234),(2345),(2346),(235),(2356),(2364),(236),(243),(2463), \\
(24),(24)(56),(24356),(24635),(245)(36),(2543),(254),(2564), \\
(2546),(25)(364),(263),(26345),(26435),(2635) \\
\varepsilon,(123)(456),(134652),(142536),(154)(263),(162435)\end{array}$ \\
\hline & \multirow{3}{*}{120} & Aut $Q$ & $\{\varepsilon\}$ \\
\hline \multirow[t]{2}{*}{6} & & AAut $\bar{Q}$ & $\varnothing$ \\
\hline & & $\langle I(Q)\rangle$ & $\begin{array}{l}(56),(45),(456),(465),(46),(3465),(3564),(35),(3546),(364), \\
(3645),(23)(56),(23)(45),(2345),(235)(46),(243),(24),(2465), \\
(246),(2543),(2564),(256),(25)(46),(2654),(264),(26),(2645)\end{array}$ \\
\hline \multirow[b]{4}{*}{7} & \multirow[b]{4}{*}{120} & $Q_{L}$ & $\varepsilon,(123)(456),(132465),(142536),(152634),(162)(354)$ \\
\hline & & Aut $Q$ & $\{\varepsilon\}$ \\
\hline & & AAut $Q$ & $\varnothing$ \\
\hline & & $\langle I(Q)\rangle$ & $\begin{array}{l}\text { (56), (456), (465), (346), (354), (3546), (36), (3645), (23)(56), } \\
(23)(45),(2346),(2356),(2465),(24356),(24635),(24)(36), \\
(245)(36),(254),(2564),(25),(2546),(254)(36),(25436),(25)(36), \\
(2536),(2653),(263)(45),(264),(2645),(2634),(26345)\end{array}$ \\
\hline \multirow[b]{4}{*}{8} & \multirow[b]{4}{*}{120} & $Q_{L}$ & $\varepsilon,(123)(456),(132465),(143526),(1542)(36),(162534)$ \\
\hline & & Aut $Q$ & $\{\varepsilon\}$ \\
\hline & & AAut $Q$ & $\varnothing$ \\
\hline & & $\langle I(Q)\rangle$ & $\begin{array}{l}(456),(465),(3465),(35),(3546),(364),(3645),(23),(23)(56), \\
(23)(45),(2346),(2356),(235)(46),(2365),(243),(24),(246), \\
(24536),(25643),(2563),(2564),(25),(25)(46),(2546),(25)(346), \\
(25346),(25)(36),(2536),(264),(2645),(26345),(26)(35)\end{array}$ \\
\hline
\end{tabular}




\begin{tabular}{|c|c|c|c|}
\hline № & $\overline{\mathrm{CL}}$ & & Класс изотопии IV \\
\hline \multirow[b]{4}{*}{9} & \multirow[b]{4}{*}{120} & $Q_{L}$ & $\varepsilon,(123)(456),(135)(246),(142536),(152634),(165432)$ \\
\hline & & Aut $Q$ & $\{\varepsilon\}$ \\
\hline & & AAut $Q$ & $\varnothing$ \\
\hline & & $\langle I(Q)\rangle$ & $\begin{array}{l}(56),(456),(465),(345),(3456),(346),(354),(3564),(3546), \\
(3654),(36),(234),(2346),(2356),(2364),(24),(245),(2435), \\
(24635),(24)(36),(245)(36),(253),(2563),(254),(25),(25364), \\
(254)(36),(25436),(25)(36),(2653),(264),(26)(45),(26345), \\
(26)(35)\end{array}$ \\
\hline \multirow[b]{4}{*}{10} & \multirow[b]{4}{*}{120} & $Q_{L}$ & $\varepsilon,(123)(456),(1365)(24),(143526),(154632),(162534)$ \\
\hline & & Aut $Q$ & $\{\varepsilon\}$ \\
\hline & & AAut $Q$ & $\varnothing$ \\
\hline & & $\langle I(\bar{Q})\rangle$ & $\begin{array}{l}(456),(465),(3465),(346),(35),(364),(3645),(23),(23)(456), \\
(23)(465),(2345),(235),(2356),(2365),(236),(243),(2453),(24), \\
(2456),(24)(35),(2436),(24536),(2543),(25643),(2563),(25463), \\
(256),(25)(46),(2546),(25634),(25)(34),(25346),(25)(36), \\
(2643),(263),(26),(26435),(26)(35)\end{array}$ \\
\hline
\end{tabular}

\begin{tabular}{||c|c|c|l||}
\hline \hline № & CL & \multicolumn{2}{|c||}{ Класс изотопии V } \\
\hline \multirow{3}{*}{1} & \multirow{3}{*}{120} & $Q_{L}$ & $\varepsilon,(12)(34)(56),(13)(25)(46),(145)(263),(154)(236),(16)(24)(35)$ \\
\cline { 3 - 4 } & & Aut $Q$ & $\{\varepsilon,(36)(45),(23)(45),(236),(263),(26)(45)\} \cong S_{3}$ \\
\cline { 2 - 4 } & & AAut $Q$ & $(45),(36),(23),(236)(45),(263)(45),(26)$ \\
\cline { 2 - 4 } & & $\langle I(Q)\rangle$ & $(36)(45),(23)(45),(236),(263),(26)(45)$ \\
\hline
\end{tabular}

\begin{tabular}{|c|c|c|c|}
\hline № & $\overline{\mathrm{CL}}$ & & Класс изотопии VI \\
\hline \multirow{4}{*}{1} & \multirow{4}{*}{120} & $Q_{L}$ & $\varepsilon,(12)(34)(56),(13)(25)(46),(145)(263),(154236),(1624)(35)$ \\
\hline & & Aut $Q$ & $\{\varepsilon\}$ \\
\hline & & AAut $Q$ & (45) \\
\hline & & $\langle I(Q)\rangle$ & $\begin{array}{l}(56),(46),(34),(34)(56),(3456),(35),(35)(46),(3546),(36)(45), \\
(23)(45),(2346),(2653),(26)(45)\end{array}$ \\
\hline \multirow{4}{*}{2} & \multirow{4}{*}{120} & $Q_{L}$ & $\varepsilon,(12)(34)(56),(13)(25)(46),(145)(263),(153624),(16)(2354)$ \\
\hline & & Aut $Q$ & $\{\varepsilon\}$ \\
\hline & & AAut $Q$ & (26) \\
\hline & & $\langle I(Q)\rangle$ & $\begin{array}{l}(56),(34),(34)(56),(35)(46),(3546),(3645),(2354),(236), \\
(24)(35),(25),(25)(34),(263),(26)(45)\end{array}$ \\
\hline \multirow{4}{*}{3} & \multirow{4}{*}{120} & $Q_{L}$ & $\varepsilon,(12)(34)(56),(13)(25)(46),(145)(263),(1536)(24),(162354)$ \\
\hline & & Aut $Q$ & $\{\varepsilon\}$ \\
\hline & & AAut $Q$ & $\varnothing$ \\
\hline & & $\langle I(Q)\rangle$ & $\begin{array}{l}(56),(34),(34)(56),(3546),(3645),(36)(45),(2346),(2354),(24), \\
(25)(46),(2546),(2653),(2645),(26)(45)\end{array}$ \\
\hline \multirow{4}{*}{4} & \multirow{4}{*}{120} & $Q_{L}$ & $\varepsilon,(12)(34)(56),(13)(2546),(145326),(152364),(1635)(24)$ \\
\hline & & Aut $Q$ & $\{\varepsilon\}$ \\
\hline & & $\overline{\text { AAut } Q}$ & $\varnothing$ \\
\hline & & $\langle I(Q)\rangle$ & $\begin{array}{l}(56),(34),(35)(46),(3546),(3645),(36)(45),(2345),(2364),(24), \\
(24)(56),(2563),(2546),(26)(45)\end{array}$ \\
\hline
\end{tabular}




\begin{tabular}{||c|c|c|l||}
\hline \hline № & CL & \multicolumn{1}{|c||}{ Класс изотопии VI } \\
\hline \multirow{3}{*}{5} & \multirow{5}{*}{60} & $Q_{L}$ & $\varepsilon,(12)(34)(56),(1325)(46),(145263),(154236),(1624)(35)$ \\
\cline { 2 - 4 } & & Aut $Q$ & $\{\varepsilon,(36)(45)\} \cong Z_{2}$ \\
\cline { 2 - 4 } & & AAut $Q$ & $(45),(36)$ \\
\cline { 2 - 4 } & & $\langle I(Q)\rangle$ & $\begin{array}{l}(56),(46),(34),(3456),(35),(3546),(3654),(3645),(36)(45), \\
(23)(45),(236),(263),(26)(45)\end{array}$ \\
\hline
\end{tabular}

\begin{tabular}{|c|c|c|c|}
\hline № & $\mathrm{CL}$ & & Класс изотопии VII \\
\hline \multirow{4}{*}{1} & \multirow{4}{*}{60} & $Q_{L}$ & $\varepsilon,(12)(34)(56),(13)(25)(46),(145326),(15)(24)(36),(162354)$ \\
\hline & & Aut $Q$ & $\{\varepsilon,(25)(46)\} \cong Z_{2}$ \\
\hline & & AAut $Q$ & $\varnothing$ \\
\hline & & $\langle I(Q)\rangle$ & $\begin{array}{l}(56),(45),(34),(34)(56),(354),(356),(36),(3645),(2346),(235), \\
(243),(24),(24)(36),(253),(2546),(263),(26),(2645),(26)(45)\end{array}$ \\
\hline \multirow{4}{*}{2} & \multirow{4}{*}{120} & $Q_{L}$ & $\varepsilon,(12)(34)(56),(13)(2546),(145)(263),(1536)(24),(164)(235)$ \\
\hline & & Aut $Q$ & $\{\varepsilon\}$ \\
\hline & & AAut $Q$ & $\varnothing$ \\
\hline & & $\langle I(Q)\rangle$ & $\begin{array}{l}(56),(45),(465),(34),(34)(56),(346),(36),(3645),(23)(46), \\
(2354),(243)(56),(24),(24)(56),(2465),(25)(46),(2546),(2653), \\
(2645),(26)(45),(26)(34),(26)(345)\end{array}$ \\
\hline \multirow{4}{*}{3} & \multirow{4}{*}{60} & $Q_{L}$ & $\varepsilon,(12)(34)(56),(1325)(46),(1426)(35),(154)(236),(163)(245)$ \\
\hline & & Aut $Q$ & $\{\varepsilon,(34)(56)\} \cong Z_{2}$ \\
\hline & & AAut $Q$ & $\varnothing$ \\
\hline & & $\langle I(Q)\rangle$ & $\begin{array}{l}(45),(46),(34)(56),(3456),(35),(35)(46),(3654),(36),(36)(45), \\
(234),(2365),(236)(45),(243),(2456),(245)(36),(2563), \\
(25)(346),(25)(36),(2654),(26)(45),(26)(354)\end{array}$ \\
\hline \multirow{4}{*}{4} & \multirow{4}{*}{120} & $Q_{L}$ & $\varepsilon,(12)(3456),(1325)(46),(143)(265),(154236),(1624)(35)$ \\
\hline & & Aut $Q$ & $\{\varepsilon\}$ \\
\hline & & AAut $Q$ & $\varnothing$ \\
\hline & & $\langle I(Q)\rangle$ & $\begin{array}{l}(46),(34),(34)(56),(3456),(35),(35)(46),(364),(3645),(36)(45), \\
(23456),(236),(243),(24),(24)(56),(2543),(25)(34),(263), \\
(2654),(26)(45),(26354)\end{array}$ \\
\hline
\end{tabular}

\begin{tabular}{|c|c|c|c|}
\hline № & $\mathrm{CL}$ & \multicolumn{2}{|r|}{ Класс изотопии VIII } \\
\hline \multirow{4}{*}{1} & \multirow{4}{*}{6} & $Q_{L}$ & $\begin{array}{llll}\varepsilon, \quad(12)(34)(56), & (13)(25)(46), & (14)(26)(35), & (15)(24)(36), \\
(16)(23)(45) & \end{array}$ \\
\hline & & Aut $Q$ & $\begin{array}{l}\{\varepsilon,(34)(56),(3546),(3645),(23)(45),(2346),(23564),(2365), \\
(24653),(2456),(2435),(24)(36),(2563),(25)(46),(2534), \\
(25436),(2643),(2654),(26345),(26)(35)\} \cong Z_{5} \lambda Z_{4}\end{array}$ \\
\hline & & AAut $Q$ & $\varnothing$ \\
\hline & & $\langle I(Q)\rangle$ & $\begin{array}{l}(456),(465),(34)(56),(345),(3456),(3465),(346),(354),(3564), \\
(356),(3654),(364),(365),(3645),(23)(45),(234),(2345),(2346), \\
(2354),(235),(2356),(2364),(236),(243),(2453),(2463),(245), \\
(2465),(246),(2435),(24)(36),(2436),(2543),(253),(2563),(254), \\
(2564),(256),(25)(46),(2546),(2536),(2653),(263),(2654),(264), \\
(265),(2645),(2634),(2635),(26)(35)\end{array}$ \\
\hline
\end{tabular}




\begin{tabular}{|c|c|c|c|}
\hline № & CL & & Класс изотопии VIII \\
\hline \multirow[b]{4}{*}{2} & \multirow[b]{4}{*}{30} & $Q_{L}$ & $\varepsilon,(12)(34)(56),(136)(254),(145)(263),(153)(246),(164)(235)$ \\
\hline & & Aut $Q$ & $\{\varepsilon,(34)(56),(3546),(3645)\} \cong Z_{4}$ \\
\hline & & AAut $Q$ & $\varnothing$ \\
\hline & & $\langle I(Q)\rangle$ & $\begin{array}{l}(456),(465),(34)(56),(345),(3456),(3465),(346),(354), \\
(3564),(356),(3654),(364),(365),(3645),(23)(56),(23)(46), \\
(234),(23456),(23546),(2364),(23645),(243),(2453),(24)(56), \\
(24)(35),(24635),(24365),(24536),(25643),(25463),(256), \\
(25)(34),(25364),(25)(36),(2536),(26453),(265),(2645), \\
(26)(45),(26534),(26)(34),(26354)\end{array}$ \\
\hline
\end{tabular}

\begin{tabular}{|c|c|c|c|}
\hline № & CL & & Класс изотопии IX \\
\hline \multirow{4}{*}{1} & \multirow{4}{*}{60} & $Q_{L}$ & $\varepsilon,(12)(34)(56),(13)(2546),(145326),(15)(2364),(163524)$ \\
\hline & & Aut $Q$ & $\{\varepsilon,(35)(46)\} \cong Z_{2}$ \\
\hline & & AAut $Q$ & $\varnothing$ \\
\hline & & $\langle I(Q)\rangle$ & $\begin{array}{l}(56),(45),(34),(3546),(36),(3645),(36)(45),(234),(235),(2364), \\
(236),(24),(24)(56),(253),(254),(256),(2546),(26),(26)(34)\end{array}$ \\
\hline \multirow{4}{*}{2} & \multirow{4}{*}{120} & $Q_{L}$ & $\varepsilon,(12)(34)(56),(13)(2546),(145326),(1524)(36),(164235)$ \\
\hline & & Aut $Q$ & $\{\varepsilon\}$ \\
\hline & & AAut $Q$ & $\varnothing$ \\
\hline & & $\langle I(Q)\rangle$ & $\begin{array}{l}(56),(45),(465),(34),(34)(56),(3465),(35)(46),(3546),(3645), \\
(36)(45),(23)(46),(234)(56),(2345),(24),(24)(56),(246), \\
(24)(36),(245)(36),(2563),(2546),(26)\end{array}$ \\
\hline \multirow{4}{*}{3} & \multirow{4}{*}{120} & $Q_{L}$ & $\varepsilon,(12)(34)(56),(1325)(46),(145263),(1536)(24),(162354)$ \\
\hline & & Aut $Q$ & $\{\varepsilon\}$ \\
\hline & & AAut $Q$ & $\varnothing$ \\
\hline & & $\langle I(Q)\rangle$ & $\begin{array}{l}(56),(34),(34)(56),(35),(35)(46),(3546),(3654),(365),(3645), \\
(36)(45),(2354),(23546),(236),(24)(35),(253),(25),(25)(46), \\
(263),(2645),(26)(45),(26345)\end{array}$ \\
\hline \multirow{4}{*}{4} & \multirow{4}{*}{60} & $Q_{L}$ & $\varepsilon,(12)(34)(56),(132546),(1453)(26),(152364),(1635)(24)$ \\
\hline & & Aut $Q$ & $\{\varepsilon,(35)(46)\} \cong Z_{2}$ \\
\hline & & AAut $Q$ & $\varnothing$ \\
\hline & & $\langle I(Q)\rangle$ & $\begin{array}{l}(56),(45),(34),(34)(56),(35)(46),(3546),(36),(3645),(36)(45), \\
(235),(2364),(236)(45),(24)(356),(24)(36),(253),(2546), \\
(254)(36),(26)(45),(26)(345)\end{array}$ \\
\hline
\end{tabular}

\begin{tabular}{||l|l|l|l||}
\hline \hline № & CL & \multicolumn{2}{|c||}{ Класс изотопии X } \\
\hline \multirow{5}{*}{1} & & $Q_{L}$ & $\varepsilon,(12)(34)(56),(13)(2546),(14)(2635),(15)(2364),(16)(2453)$ \\
\cline { 3 - 4 } & & Aut $Q$ & $\{\varepsilon,(34)(56),(35)(46),(36)(45)\} \cong K_{4}$ \\
\cline { 2 - 4 } & AAut $Q$ & $(45),(3465),(3564),(36)$ \\
\cline { 2 - 4 } & & $\langle I(Q)\rangle$ & $(45),(3465),(3564),(36),(36)(45),(23)(56),(23)(45),(23)(456)$, \\
& & $(23)(465),(23)(46),(23465),(23564),(236),(24653),(24)(56)$, \\
& & $(245),(24)(35),(24)(356),(24356),(24)(365),(24)(36),(25643)$, \\
& & & $(254),(25)(46),(25)(34),(25)(346),(25346),(25)(364),(25)(36)$, \\
& & & $(263),(26)(45),(26534),(26)(34),(26)(345),(26435),(26)(354)$, \\
\hline
\end{tabular}




\begin{tabular}{|c|c|c|c|}
\hline № & $\mathrm{CL}$ & & Класс изотопии $\mathrm{X}$ \\
\hline \multirow[b]{4}{*}{2} & \multirow[b]{4}{*}{120} & $Q_{L}$ & $\varepsilon,(12)(34)(56),(13)(2546),(1426)(35),(1524)(36),(1645)(23)$ \\
\hline & & Aut $Q$ & $\{\varepsilon\}$ \\
\hline & & AAut $Q$ & $\varnothing$ \\
\hline & & $\langle I(Q)\rangle$ & $\begin{array}{l}(56),(45),(465),(46),(34),(34)(56),(345),(3456),(3465), \\
(346),(354),(3564),(35),(3654),(36),(3645),(36)(45),(23)(56), \\
(23)(46),(234),(2364),(2365),(236),(2463),(24)(56),(245), \\
(246),(24635),(2563),(253)(46),(254),(25)(46),(25346), \\
(25436),(263),(26),(26)(45),(265)(34),(26)(354)\end{array}$ \\
\hline \multirow[b]{4}{*}{3} & \multirow[b]{4}{*}{120} & $Q_{L}$ & $\varepsilon,(12)(34)(56),(132546),(145263),(153624),(164235)$ \\
\hline & & Aut $Q$ & $\{\varepsilon\}$ \\
\hline & & AAut $Q$ & $\varnothing$ \\
\hline & & $\langle I(Q)\rangle$ & $\begin{array}{l}(56),(45),(465),(46),(34),(34)(56),(345),(3465),(346),(354), \\
(3564),(35),(356),(35)(46),(364),(36),(3645),(23)(46),(2354), \\
(2356),(23546),(2364),(243),(24),(245),(2465),(24)(356), \\
(253)(46),(2546),(256)(34),(25)(364),(2653),(2654),(26), \\
(2645),(26)(45),(265)(34)\end{array}$ \\
\hline \multirow[b]{4}{*}{4} & \multirow[b]{4}{*}{30} & $Q_{L}$ & $\varepsilon,(12)(34)(56),(132546),(142635),(152364),(162453)$ \\
\hline & & Aut $Q$ & $\{\varepsilon,(34)(56),(35)(46),(36)(45)\} \cong \mathbb{K}_{4}$ \\
\hline & & AAut $Q$ & $(45),(3465),(3564),(36)$ \\
\hline & & $\langle I(Q)\rangle$ & $\begin{array}{l}(45),(3465),(3564),(36),(36)(45),(23),(234),(234)(56),(235), \\
(235)(46),(2364),(2365),(236),(243),(243)(56),(2453),(24), \\
(245),(2456),(246),(246)(35),(2543),(253),(253)(46),(254), \\
(25),(256),(2546),(256)(34),(263),(264),(265),(26),(2634), \\
(265)(34),(264)(35),(2635)\end{array}$ \\
\hline \multirow[b]{4}{*}{5} & \multirow[b]{4}{*}{30} & $Q_{L}$ & $\varepsilon,(12)(34)(56),(1364)(25),(1453)(26),(1546)(23),(1635)(24)$ \\
\hline & & Aut $Q$ & $\{\varepsilon,(34)(56),(35)(46),(36)(45)\} \cong \mathbb{K}_{4}$ \\
\hline & & AAut $Q$ & $\varnothing$ \\
\hline & & $\langle I(Q)\rangle$ & $\begin{array}{l}(45),(3465),(3564),(36),(36)(45),(23),(23)(56),(23)(465), \\
(23564),(235),(235)(46),(2365),(236),(236)(45),(24653),(24), \\
(24)(56),(245),(2456),(246),(24)(356),(246)(35),(45)(36), \\
(2543),(253),(253)(46),(254),(25),(25)(34),(25346),(254)(36), \\
(25)(364),(263),(263)(45),(264),(26),(2634),(26)(34),(26)(345), \\
(264)(35),(26435)\end{array}$ \\
\hline \multirow[b]{4}{*}{6} & \multirow[b]{4}{*}{30} & $Q_{L}$ & $\varepsilon,(12)(34)(56),(136425),(145326),(154623),(163524)$ \\
\hline & & Aut $Q$ & $\{\varepsilon,(34)(56),(35)(46),(36)(45)\} \cong \mathbb{K}_{4}$ \\
\hline & & AAut $Q$ & $\varnothing$ \\
\hline & & $\langle I(Q)\rangle$ & $\begin{array}{l}(45),(3465),(3564),(36),(36)(45),(23),(23)(456),(23)(46),(234), \\
(234)(56),(23465),(2364),(236),(236)(45),(243),(243)(56), \\
(2453),(24),(245),(24)(35),(24356),(24)(365),(245)(36), \\
(25643),(254),(25),(256),(25)(46),(2546),(256)(34),(25)(346), \\
(254)(36),(263),(263)(45),(265),(26),(26534),(265)(34), \\
(26)(354),(2635),(26)(35)\end{array}$ \\
\hline
\end{tabular}




\begin{tabular}{|c|c|c|c|}
\hline № & $\overline{\mathrm{CL}}$ & & Класс изотопии $\mathbf{X}$ \\
\hline \multirow[b]{4}{*}{7} & \multirow[b]{4}{*}{120} & $Q_{L}$ & $\varepsilon,(12)(3456),(13)(2465),(1426)(35),(154)(236),(164325)$ \\
\hline & & Aut $Q$ & $\{\varepsilon\}$ \\
\hline & & AAut $Q$ & $(56)$ \\
\hline & & $\langle I(Q)\rangle$ & $\begin{array}{l}(45),(456),(465),(46),(34)(56),(345),(3456),(3465),(346), \\
(35)(46),(36)(45),(23),(23)(56),(23456),(23465),(235)(46), \\
(2364),(236)(45),(2453),(24),(2543),(253),(254),(25),(256), \\
(25)(46),(256)(34),(25)(364),(25)(36),(2536),(2643),(263), \\
(264),(265),(26),(26)(45),(265)(34),(26)(354),(2635),(26)(35)\end{array}$ \\
\hline \multirow[b]{4}{*}{8} & \multirow[b]{4}{*}{120} & $Q_{L}$ & $\varepsilon,(12)(3456),(132465),(1426)(35),(154)(236),(1643)(25)$ \\
\hline & & Aut $Q$ & $\{\varepsilon\}$ \\
\hline & & AAut $Q$ & $(56)$ \\
\hline & & $\langle I(Q)\rangle$ & $\begin{array}{l}(45),(456),(465),(46),(34)(56),(3564),(35)(46),(3654),(36)(45), \\
(23)(56),(23)(45),(23)(46),(234),(2345),(23456),(23465), \\
(2346),(2356),(235)(46),(2365),(236)(45),(243),(2453), \\
(24563),(24653),(2463),(24),(253)(46),(25),(256),(25346), \\
(254)(36),(25)(36),(263)(45),(265),(26),(26345),(264)(35), \\
(26)(35)\end{array}$ \\
\hline \multirow[b]{4}{*}{9} & \multirow[b]{4}{*}{120} & $Q_{L}$ & $\varepsilon,(12)(3456),(135)(246),(14)(2653),(154236),(1643)(25)$ \\
\hline & & Aut $Q$ & $\{\varepsilon\}$ \\
\hline & & AAut $Q$ & $\varnothing$ \\
\hline & & $\langle I(Q)\rangle$ & $\begin{array}{l}(56),(456),(465),(34),(34)(56),(345),(35),(35)(46),(365),(36), \\
(36)(45),(23),(23)(56),(234),(23465),(2354),(2364),(2365), \\
(243),(2453),(24)(35),(246)(35),(24)(36),(253),(2563),(254), \\
(25),(256),(25346),(25)(364),(25)(36),(2536),(2653),(26453), \\
(265),(26),(265)(34),(2635)\end{array}$ \\
\hline \multirow[b]{4}{*}{10} & \multirow[b]{4}{*}{120} & $Q_{L}$ & $\varepsilon,(12)(3456),(1325)(46),(143526),(154)(236),(1653)(24)$ \\
\hline & & Aut $Q$ & $\{\varepsilon\}$ \\
\hline & & AAut $Q$ & $\varnothing$ \\
\hline & & $\langle I(Q)\rangle$ & $\begin{array}{l}(56),(45),(456),(46),(34),(34)(56),(345),(3456),(3465),(346), \\
(354),(3564),(35),(356),(35)(46),(365),(36),(23)(56),(2345), \\
(23456),(2346),(2365),(243)(56),(2456),(246)(35),(24)(365), \\
(2543),(253),(254),(2564),(25),(25)(346),(2654),(26),(2645), \\
(26)(45),(264)(35)\end{array}$ \\
\hline \multirow[b]{4}{*}{11} & \multirow[b]{4}{*}{120} & $Q_{L}$ & $\varepsilon,(123)(456),(1365)(24),(1462)(35),(154326),(1634)(25)$ \\
\hline & & Aut $Q$ & $\{\varepsilon\}$ \\
\hline & & AAut $Q$ & $\varnothing$ \\
\hline & & $\langle I(Q)\rangle$ & $\begin{array}{l}(45),(456),(46),(34),(3465),(346),(354),(35)(46),(364), \\
(36),(3645),(23),(23)(56),(23)(45),(23)(456),(23)(46),(234), \\
(234)(56),(2365),(236),(23645),(24)(56),(2456),(246),(24)(35), \\
(24356),(246)(35),(2543),(25643),(2563),(253)(46),(2546), \\
(25)(34),(25)(346),(25436),(26543),(263),(26453),(263)(45), \\
(26),(2645),(26)(45),(26)(354)\end{array}$ \\
\hline
\end{tabular}




\begin{tabular}{||c|c|c|l||}
\hline \hline № & CL & \multicolumn{1}{|c||}{ Класс изотопии X } \\
\hline \multirow{5}{*}{12} & & $Q_{L}$ & $\varepsilon,(123)(456),(1365)(24),(1462)(35),(1526)(34),(163254)$ \\
\cline { 3 - 4 } & & Aut $Q$ & $\{\varepsilon\}$ \\
\cline { 3 - 4 } & AAut $Q$ & $\varnothing$ \\
\cline { 3 - 4 } & $\langle 20(Q)\rangle$ & $(46),(34),(35)(46),(3546),(364),(36),(3645),(23),(23)(56)$, \\
& & $(23)(456),(23)(46),(234),(234)(56),(2356),(236),(236)(45)$, \\
& & $(24)(56),(246),(24)(35),(246)(35),(2543),(25643),(253),(2563)$, \\
& & $(253)(46),(25463),(25),(25)(46),(2534),(25)(34),(25)(364)$, \\
& & $(26453),(265),(26),(2645),(26)(45),(26534),(265)(34),(26345)$, \\
\hline
\end{tabular}

\begin{tabular}{|c|c|c|c|}
\hline № & $\mathrm{CL}$ & & Класс изотопии XI \\
\hline \multirow[b]{4}{*}{1} & \multirow[b]{4}{*}{60} & $Q_{L}$ & $\varepsilon,(12)(34)(56),(13)(2546),(14)(2635),(1536)(24),(1645)(23)$ \\
\hline & & Aut $Q$ & $\{\varepsilon,(34)(56)\} \cong Z_{2}$ \\
\hline & & AAut $Q$ & $\varnothing$ \\
\hline & & $\langle I(Q)\rangle$ & $\begin{array}{l}(56),(456),(34),(34)(56),(345),(3456),(3465),(346),(354), \\
(3564),(3654),(364),(365),(3645),(23),(23)(56),(23)(465), \\
(23)(46),(2354),(23654),(236),(23645),(24563),(2463),(24), \\
(24)(56),(245),(24)(35),(24)(356),(24536),(2543),(254), \\
(25)(46),(2546),(25)(34),(254)(36),(25)(364),(25)(36),(263), \\
(263)(45),(26)(45),(2634),(26)(34),(26)(345),(2635),(26)(35)\end{array}$ \\
\hline \multirow{6}{*}{2} & \multirow[b]{4}{*}{30} & $Q_{L}$ & $\varepsilon,(12)(34)(56),(1325)(46),(1426)(35),(1524)(36),(1623)(45)$ \\
\hline & & Aut $Q$ & $\{\varepsilon,(34)(56),(3546),(3645)\} \cong Z_{4}$ \\
\hline & & AAut $Q$ & $\varnothing$ \\
\hline & & $\langle I(Q)\rangle$ & $\begin{array}{l}(456),(465),(34)(56),(345),(3456),(3465),(346),(354),(3564), \\
(356),(3654),(364),(365),(3645),(23)(56),(234),(2346), \\
(2354),(235),(235)(46),(23546),(23654),(2364),(243),(2453), \\
(24563),(2463),(24)(56),(246),(2435),(24635),(246)(35), \\
(2563),(25463),(254),(256),(2546),(25)(34),(25346),(254)(36), \\
(2536),(263),(263)(45),(2654),(265),(2645),(26)(34),(26354), \\
(26435),(2635) \\
\varepsilon,(12)(34)(56),(132546),(142635),(153624),(164523)\end{array}$ \\
\hline & \multirow[b]{3}{*}{60} & Aut $Q$ & $\{\varepsilon,(34)(56)\} \cong Z_{2}$ \\
\hline & & AAut $Q$ & $\varnothing$ \\
\hline 3 & & $\langle I(Q)\rangle$ & $\begin{array}{l}(56),(456),(34),(34)(56),(345),(3456),(3465),(3564),(3546), \\
(3654),(364),(365),(3645),(23),(2354),(235),(2364),(2365), \\
(2453),(2463),(24),(2456),(246),(2563),(254),(2546),(25)(34), \\
(25)(36),(2536),(263),(2654),(2645),(26)(45),(26)(34),(2635)\end{array}$ \\
\hline \multirow[b]{4}{*}{4} & \multirow[b]{4}{*}{30} & $Q_{L}$ & $\varepsilon,(12)(34)(56),(136254),(145263),(153246),(164235)$ \\
\hline & & Aut $Q$ & $\{\varepsilon,(34)(56),(3546),(3645)\} \cong Z_{4}$ \\
\hline & & AAut $Q$ & $\varnothing$ \\
\hline & & $\langle I(Q)\rangle$ & $\begin{array}{l}(456),(465),(34)(56),(345),(3456),(3465),(346),(354),(3564), \\
(356),(3654),(364),(365),(3645),(23),(23)(45),(234),(2345), \\
(2346),(2354),(2356),(2364),(236),(243),(2453),(2463),(24), \\
(245),(2465),(2435),(24)(36),(2436),(2543),(253),(2563), \\
(2564),(25),(256),(25)(46),(2546),(2536),(2653),(2654),(264), \\
(265),(26),(2645),(2634),(2635),(26)(35)\end{array}$ \\
\hline
\end{tabular}




\begin{tabular}{|c|c|c|c|}
\hline № & $\mathrm{CL}$ & & Класс изотопии XI \\
\hline & & $Q_{L}$ & $\varepsilon,(12)(3456),(135)(246),(143)(265),(154236),(164)(253)$ \\
\hline & & Aut $Q$ & $\{\varepsilon\}$ \\
\hline & & AAut $Q$ & $\varnothing$ \\
\hline 5 & 120 & $\langle I(Q)\rangle$ & $\begin{array}{l}(45),(456),(465),(46),(345),(3456),(346),(35)(46),(364), \\
(36)(45),(23)(45),(23)(465),(23)(46),(2345),(2354),(23564), \\
(2356),(2364),(2365),(236),(243),(24),(2456),(24)(35),(24635), \\
(24)(36),(2436),(245)(36),(2563),(254),(2564),(2546),(25346), \\
(25)(364),(2643),(2653),(263),(263)(45),(26),(26)(345), \\
(26354),(26435),(2635) \\
\varepsilon,(123)(456),(135)(246),(142)(365),(154326),(1634)(25)\end{array}$ \\
\hline & & Aut $Q$ & $\{\varepsilon\}$ \\
\hline & & AAut $Q$ & $\varnothing$ \\
\hline 6 & 120 & $\langle I(Q)\rangle$ & $\begin{array}{l}(345),(3456),(3465),(346),(354),(3564),(3654),(364),(36), \\
(3645),(36)(45),(23)(45),(23)(46),(234)(56),(23456),(2354), \\
(23564),(2356),(23654),(2364),(2365),(236),(243),(2463),(24), \\
(2456),(24536),(2543),(256),(2534),(25634),(25364),(25436), \\
(25)(36),(2536),(26453),(26),(26)(45),(26)(34),(26354), \\
(26435),(26)(354),(2635),(26)(35)\end{array}$ \\
\hline \multirow[b]{4}{*}{7} & \multirow[b]{4}{*}{120} & $Q_{L}$ & $\varepsilon,(123)(456),(135)(246),(142)(365),(1526)(34),(163254)$ \\
\hline & & Aut $Q$ & $\{\varepsilon\}$ \\
\hline & & AAut $Q$ & $\varnothing$ \\
\hline & & $\langle I(Q)\rangle$ & $\begin{array}{l}(45),(346),(354),(35),(35)(46),(364),(36),(3645),(36)(45), \\
(23)(46),(234),(2346),(2354),(235),(2356),(23654),(2364), \\
(236),(243),(24)(56),(24)(356),(2436),(245)(36),(2543),(253), \\
(2563),(253)(46),(254),(25),(256),(25634),(25)(34),(25)(364), \\
(25)(36),(26),(2645),(26)(34),(26354),(26)(354),(2635)\end{array}$ \\
\hline
\end{tabular}

\begin{tabular}{|c|c|c|c|}
\hline № & $\mathrm{CL}$ & & Класс изотопии XII \\
\hline \multirow[b]{4}{*}{1} & \multirow[b]{4}{*}{60} & $Q_{L}$ & $\varepsilon,(12)(34)(56),(13)(2546),(142635),(152364),(16)(2453)$ \\
\hline & & Aut $Q$ & $\{\varepsilon,(36)(45)\} \cong Z_{2}$ \\
\hline & & AAut $Q$ & $(45),(36)$ \\
\hline & & $\langle I(Q)\rangle$ & $\begin{array}{l}(34)(56),(35)(46),(36)(45),(23),(234)(56),(235)(46),(236), \\
(243),(2453),(24653),(24)(56),(245),(2456),(246),(24)(35), \\
(24)(356),(24356),(24)(365),(24)(36),(2543),(25643),(253), \\
(254),(256),(25)(46),(2546),(25)(34),(25)(346),(25346), \\
(25)(364),(25)(36),(263),(26),(265)(34),(264)(35)\end{array}$ \\
\hline \multirow[b]{4}{*}{2} & \multirow[b]{4}{*}{120} & $Q_{L}$ & $\varepsilon,(12)(34)(56),(13)(2546),(1426)(35),(152364),(163245)$ \\
\hline & & Aut $Q$ & $\{\varepsilon\}$ \\
\hline & & AAut $Q$ & (45) \\
\hline & & $\langle I(Q)\rangle$ & $\begin{array}{l}(45),(456),(465),(34),(345),(3456),(346),(354),(35),(356), \\
(3546),(3654),(36),(3645),(36)(45),(23),(234),(235),(2364), \\
(243)(56),(24)(56),(245),(2456),(24536),(2563),(253)(46), \\
(254),(25)(46),(2546),(25436),(26)(34),(26)(345),(26)(354), \\
(26)(35)\end{array}$ \\
\hline
\end{tabular}




\begin{tabular}{|c|c|c|c|}
\hline № & $\mathrm{CL}$ & & Класс изотопии XII \\
\hline \multirow[b]{4}{*}{3} & \multirow[b]{4}{*}{120} & $Q_{L}$ & $\varepsilon,(12)(34)(56),(1325)(46),(1453)(26),(154236),(163524)$ \\
\hline & & Aut $Q$ & $\{\varepsilon\}$ \\
\hline & & AAut $Q$ & (45) \\
\hline & & $\langle I(Q)\rangle$ & $\begin{array}{l}(56),(45),(456),(465),(46),(345),(3456),(354),(3546),(3654), \\
(364),(365),(36),(3645),(36)(45),(23)(45),(23654),(2364), \\
(2365),(23645),(24),(246),(24)(35),(24)(356),(2563),(25), \\
(256),(25)(34),(25)(346),(26)(45),(265)(34),(26)(34),(264)(35), \\
(26)(35)\end{array}$ \\
\hline \multirow[b]{4}{*}{4} & \multirow[b]{4}{*}{60} & $Q_{L}$ & $\varepsilon,(12)(34)(56),(1364)(25),(145326),(154623),(1635)(24)$ \\
\hline & & Aut $Q$ & $\{\varepsilon,(36)(45)\} \cong Z_{2}$ \\
\hline & & AAut $Q$ & $(45),(36)$ \\
\hline & & $\langle I(Q)\rangle$ & 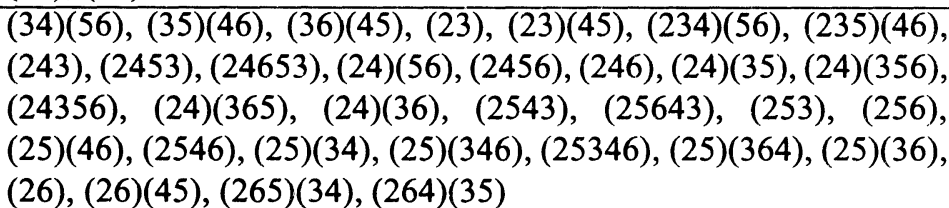 \\
\hline
\end{tabular}

\begin{tabular}{|c|c|c|c|}
\hline № & $\overline{\mathrm{CL}}$ & & Класс изотопии XIII \\
\hline \multirow{4}{*}{1} & \multirow{4}{*}{6} & $Q_{L}$ & $\varepsilon,(12)(3456),(13)(2465),(14)(2536),(15)(2643),(16)(2354)$ \\
\hline & & Aut $Q$ & $\begin{array}{l}\{\varepsilon,(3456),(35)(46),(3654),(23)(56),(2346),(2354),(23645), \\
(2453),(2465),(24356),(24)(36),(25463),(2564),(25)(34), \\
(2536),(2643),(26)(45),(26534),(2635)\} \cong Z_{5} \lambda Z_{4}\end{array}$ \\
\hline & & AAut $Q$ & $=$ Aut $Q \cong Z_{5} \lambda Z_{4}$ \\
\hline & & $\langle I(Q) \overline{\langle}$ & $\begin{array}{l}(34)(56),(35)(46),(36)(45),(23)(56),(23)(45),(23)(46),(24)(56), \\
(24)(35),(24)(36),(25)(46),(25)(34),(25)(36),(26)(45),(26)(34), \\
(26)(35)\end{array}$ \\
\hline \multirow[b]{4}{*}{2} & \multirow[b]{4}{*}{30} & $Q_{L}$ & $\varepsilon,(12)(3456),(1354)(26),(1465)(23),(1536)(24),(1643)(25)$ \\
\hline & & Aut $Q$ & $\{\varepsilon,(3456),(35)(46),(3654)\} \cong Z_{4}$ \\
\hline & & AAut $Q$ & $(46),(34)(56),(35),(36)(45)$ \\
\hline & & $\langle I(Q)\rangle$ & $\begin{array}{l}(34)(56),(35)(46),(36)(45),(23)(46),(234),(23456),(23564), \\
(235),(23546),(23654),(236),(243),(24563),(24653),(245), \\
(246),(24)(35),(24635),(24365),(253),(254),(256),(25)(46), \\
(25634),(25346),(25364),(25436),(26543),(263),(26453),(264),\end{array}$ \\
\hline
\end{tabular}

\begin{tabular}{|c|c|c|c|}
\hline № & $\mathrm{CL}$ & \multicolumn{2}{|r|}{$\overline{\text { Класс изотопии XIV }}$} \\
\hline \multirow{4}{*}{1} & \multirow{4}{*}{60} & $Q_{L}$ & $\varepsilon,(12)(3456),(13)(2465),(14)(2536),(154326),(164235)$ \\
\hline & & Aut $Q$ & $\{\varepsilon,(23)(56)\} \cong Z_{2}$ \\
\hline & & AAut $Q$ & $=$ Aut $Q \cong Z_{2}$ \\
\hline & & $\langle I(Q)\rangle$ & $\begin{array}{l}(45),(46),(34),(345),(346),(35)(46),(36)(45),(23)(56),(23)(45), \\
(23)(46),(24),(245),(246),(2435),(25)(46),(2536),(26)(45), \\
(2634)\end{array}$ \\
\hline
\end{tabular}




\begin{tabular}{|c|c|c|c|}
\hline № & $\overline{\mathrm{CL}}$ & & 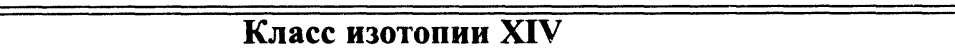 \\
\hline \multirow{4}{*}{2} & \multirow{4}{*}{30} & $Q_{L}$ & $\varepsilon,(12)(3456),(132465),(142536),(152643),(162354)$ \\
\hline & & Aut $Q$ & $\{\varepsilon,(3456),(35)(46),(3654)\} \cong Z_{4}$ \\
\hline & & AAut $Q$ & $=$ Aut $Q \cong Z_{4}$ \\
\hline & & $\langle I(Q)\rangle$ & $\begin{array}{l}(34)(56),(35)(46),(36)(45),(23),(23)(46),(234),(2345),(236), \\
(243),(24),(245),(2456),(24)(35),(2563),(254),(25),(256), \\
(25)(46),(263),(265),(26),(2634),(26)(35)\end{array}$ \\
\hline \multirow[b]{4}{*}{3} & \multirow[b]{4}{*}{120} & $Q_{L}$ & $\varepsilon,(12)(3456),(132654),(146235),(1536)(24),(1643)(25)$ \\
\hline & & Aut $Q$ & $\{\varepsilon\}$ \\
\hline & & AAut $Q$ & (46) \\
\hline & & $\langle I(Q)\rangle$ & $\begin{array}{l}(56),(45),(456),(465),(34)(56),(345),(35),(35)(46),(365), \\
(36)(45),(23)(46),(2345),(2346),(23564),(235),(2356),(23546), \\
(2364),(2365),(2453),(2463),(245),(2465),(24)(35),(24635), \\
(246)(35),(24)(365),(253),(254),(256),(25)(46),(256)(34), \\
(254)(36),(2643),(265),(2645),(26)(345),(264)(35),(26435), \\
(26)(35)\end{array}$ \\
\hline \multirow[b]{4}{*}{4} & \multirow[b]{4}{*}{60} & $Q_{L}$ & $\varepsilon,(12)(3456),(1354)(26),(146523),(1536)(24),(164325)$ \\
\hline & & Aut $Q$ & $\{\varepsilon,(35)(46)\} \cong Z_{2}$ \\
\hline & & AAut $Q$ & $(34)(56),(36)(45)$ \\
\hline & & $\langle I(Q)\rangle$ & $\begin{array}{l}(3456),(35)(46),(3654),(23),(23)(456),(2345),(2346),(2356), \\
(235)(46),(23546),(23654),(236)(45),(24563),(24),(2465), \\
(2435),(24635),(246)(35),(24)(365),(2436),(245)(36),(2563), \\
(253)(46),(2564),(25),(2534),(25)(346),(25364),(254)(36), \\
(25436),(2643),(2653),(26453),(263)(45),(2654),(26),(26345), \\
(264)(35),(26)(354)\end{array}$ \\
\hline \multirow[b]{4}{*}{5} & \multirow[b]{4}{*}{30} & $Q_{L}$ & $\varepsilon,(12)(3456),(135426),(146523),(153624),(164325)$ \\
\hline & & Aut $Q$ & $\{\varepsilon,(3456),(35)(46),(3654)\} \cong Z_{4}$ \\
\hline & & AAut $Q$ & $(46),(34)(56),(35),(36)(45)$ \\
\hline & & $\langle I(Q)\rangle$ & $\begin{array}{l}(34)(56),(35)(46),(36)(45),(23)(56),(23)(45),(23)(46),(234)(56), \\
(23465),(2346),(235),(2364),(23645),(236)(45),(243)(56), \\
(2453),(24)(56),(246),(24)(35),(2435),(24356),(24)(36), \\
(245)(36),(24536),(25643),(253),(25463),(2564),(25)(46), \\
(2546),(25)(34),(256)(34),(254)(36),(25)(36),(2653),(263)(45), \\
(264),(26)(45),(26534),(265)(34),(26)(34),(26354),(2635), \\
(26)(35)\end{array}$ \\
\hline \multirow[b]{4}{*}{6} & \multirow[b]{4}{*}{120} & $Q_{L}$ & $\varepsilon,(1234)(56),(135246),(1453)(26),(1542)(36),(164325)$ \\
\hline & & Aut $Q$ & $\{\varepsilon\}$ \\
\hline & & AAut $Q$ & $\varnothing$ \\
\hline & & $\langle I(Q)\rangle$ & 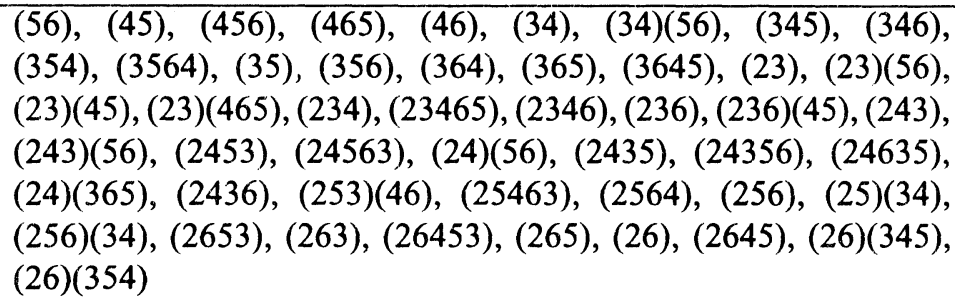 \\
\hline
\end{tabular}




\begin{tabular}{||c|c|c|l||}
\hline \hline № & CL & \multicolumn{1}{|c||}{ Класс изотопии XIV } \\
\hline \multirow{5}{*}{7} & & $Q_{L}$ & $\varepsilon,(1234)(56),(132546),(145263),(153642),(162435)$ \\
\cline { 3 - 4 } & & Aut $Q$ & $\{\varepsilon\}$ \\
\cline { 2 - 4 } & AAut $Q$ & $\varnothing$ \\
\cline { 2 - 4 } & $\langle I(Q)\rangle$ & $(45),(456),(465),(46),(346),(35),(356),(3546),(364),(365)$, \\
& & $(36),(36)(45),(23),(23)(45),(23)(456),(2346),(2354),(235)$, \\
& & $(2356),(235)(46),(23546),(23654),(236),(245),(24)(356)$, \\
& & $(24)(36),(2436),(253)(46),(254),(2564),(25),(256),(2546)$, \\
& & & $(2534),(25)(34),(25364),(254)(36),(25)(364),(25)(36),(2536)$, \\
& & & $(26543),(263),(263)(45),(265),(26),(26534),(26345),(26)(354)$ \\
\hline
\end{tabular}

\begin{tabular}{|c|c|c|c|}
\hline № & $\overline{\mathrm{CL}}$ & & Класс изотопии XV \\
\hline \multirow{4}{*}{1} & \multirow{4}{*}{120} & $Q_{L}$ & $\varepsilon,(12)(3456),(132465),(142536),(1543)(26),(164)(235)$ \\
\hline & & Aut $Q$ & $\{\varepsilon\}$ \\
\hline & & AAut $Q$ & $\{\varepsilon\}$ \\
\hline & & $\langle I(Q)\rangle$ & $\begin{array}{l}(34),(35)(46),(36)(45),(23)(46),(2345),(235),(2364),(2463), \\
(246),(2435),(253),(2564),(256),(25)(46),(2536),(263),(26)\end{array}$ \\
\hline \multirow[b]{4}{*}{2} & \multirow[b]{4}{*}{120} & $Q_{L}$ & $\varepsilon,(12)(3456),(135)(246),(1436)(25),(153264),(165423)$ \\
\hline & & Aut $Q$ & $\{\varepsilon\}$ \\
\hline & & AAut $Q$ & (35)(46) \\
\hline & & $\langle I(Q) \bar{Y}$ & $\begin{array}{l}(56),(34),(34)(56),(3456),(35)(46),(3546),(3654),(3645), \\
(234)(56),(23465),(2346),(2453),(246),(24)(35),(24)(356), \\
(24)(365),(2436),(25643),(2564),(256)(34),(2654),(264), \\
(26)(345),(26)(354),(2635),(26)(35)\end{array}$ \\
\hline \multirow{4}{*}{3} & \multirow{4}{*}{60} & $Q_{L}$ & $\varepsilon,(12)(3456),(135246),(143625),(153264),(165423)$ \\
\hline & & Aut $Q$ & Aut $Q \cong Z_{2}$ \\
\hline & & AAut $Q$ & $(35)(46)$ \\
\hline & & $\langle I(Q)\rangle$ & $\begin{array}{l}(56),(465),(34),(345),(356),(35)(46),(364),(234),(2453), \\
(2465),(246),(24)(35),(256),(2643),(264),(2635),(26)(35)\end{array}$ \\
\hline \multirow[b]{4}{*}{4} & \multirow[b]{4}{*}{120} & $Q_{L}$ & $\varepsilon,(12)(3456),(13)(2654),(146235),(152436),(164)(253)$ \\
\hline & & Aut $Q$ & $\{\varepsilon\}$ \\
\hline & & AAut $Q$ & $(46)$ \\
\hline & & $\langle I(Q)\rangle$ & $\begin{array}{l}(56),(45),(34)(56),(3456),(3465),(3564),(35)(46),(3546), \\
(3654),(3645),(36)(45),(23)(46),(2345),(2354),(235),(2356), \\
(2365),(24653),(2463),(24)(56),(2543),(253),(2563),(25)(46), \\
(2643),(26453),(26)(45)\end{array}$ \\
\hline \multirow[b]{4}{*}{5} & \multirow[b]{4}{*}{60} & $Q_{L}$ & $\varepsilon,(12)(3456),(132654),(146235),(152436),(164253)$ \\
\hline & & Aut $Q$ & $\{\varepsilon,(35)(46)\} \cong Z_{2}$ \\
\hline & & AAut $Q$ & $(46),(35)$ \\
\hline & & $\langle I(Q)\rangle$ & $\begin{array}{l}(56),(45),(456),(465),(34),(345),(346),(354),(356),(35)(46), \\
(364),(365),(36),(23)(46),(2346),(2354),(235),(2356),(2364), \\
(24653),(24635),(253),(2564),(25)(46),(2546),(2534),(2536), \\
(26453),(26435)\end{array}$ \\
\hline
\end{tabular}




\begin{tabular}{|c|c|c|c|}
\hline № & $\mathrm{CL}$ & & Класс изотопии XV \\
\hline \multirow[b]{4}{*}{6} & \multirow[b]{4}{*}{120} & $Q_{L}$ & $\varepsilon,(123)(456),(134652),(1435)(26),(1536)(24),(163254)$ \\
\hline & & Aut $Q$ & $\{\varepsilon\}$ \\
\hline & & AAut $Q$ & $(56)$ \\
\hline & & $\langle I(Q)\rangle$ & $\begin{array}{l}(456),(465),(3456),(3465),(35),(3546),(36),(3645),(23)(56), \\
(23)(45),(23)(46),(234)(56),(2345),(2346),(2356),(235)(46), \\
(2365),(236)(45),(243),(24),(24635),(24536),(2543),(253)(46), \\
(254),(256)(34),(2643),(263)(45),(264),(265)(34)\end{array}$ \\
\hline \multirow[b]{4}{*}{7} & \multirow[b]{4}{*}{120} & $Q_{L}$ & $\varepsilon,(123)(456),(135246),(1425)(36),(1534)(26),(165432)$ \\
\hline & & Aut $Q$ & $\{\varepsilon\}$ \\
\hline & & AAut $Q$ & $\varnothing$ \\
\hline & & $\langle I(Q)\rangle$ & $\begin{array}{l}(456),(465),(345),(346),(354),(3654),(365),(36),(23)(56), \\
(23)(45),(23)(46),(2345),(23456),(2346),(2354),(2356), \\
(243)(56),(245),(2456),(24635),(246)(35),(245)(36),(253), \\
(2563),(25),(256)(34),(25)(364),(25)(36),(2536),(2653), \\
(26453),(265),(26)(45),(2635),(26)(35)\end{array}$ \\
\hline \multirow[b]{4}{*}{8} & \multirow[b]{4}{*}{120} & $Q_{L}$ & $\varepsilon,(123)(456),(136524),(1426)(35),(154632),(1625)(34)$ \\
\hline & & Aut $Q$ & $\{\varepsilon\}$ \\
\hline & & AAut $Q$ & $\varnothing$ \\
\hline & & $\overline{\langle I(Q)\rangle}$ & $\begin{array}{l}(456),(465),(346),(35),(364),(365),(3645),(23)(56),(23)(45), \\
(23)(46),(2345),(2346),(2356),(2365),(23645),(243)(56), \\
(2456),(2465),(246),(2435),(24536),(253),(2563),(25463), \\
(256),(25)(46),(2534),(25)(346),(25)(36),(26),(265)(34), \\
(264)(35),(26)(354),(26)(35)\end{array}$ \\
\hline \multirow[b]{4}{*}{9} & \multirow[b]{4}{*}{120} & $Q_{L}$ & $\varepsilon,(1234)(56),(135246),(145362),(154263),(164325)$ \\
\hline & & Aut $Q$ & $\{\varepsilon\}$ \\
\hline & & AAut $Q$ & $\varnothing$ \\
\hline & & $\langle I(Q)\rangle$ & $\begin{array}{l}(56),(456),(34),(34)(56),(345),(3456),(3465),(346),(354), \\
(35)(46),(3546),(3654),(3645),(36)(45),(2354),(243),(243)(56), \\
(2463),(245),(246),(24)(35),(2435),(24635),(24)(36),(24536), \\
(253)(46),(254),(2564),(256)(34),(25)(346),(26543),(263)(45), \\
(264),(265),(2635),(26)(35)\end{array}$ \\
\hline \multirow[b]{4}{*}{10} & \multirow[b]{4}{*}{120} & $Q_{L}$ & $\varepsilon,(1234)(56),(136425),(143526),(154632),(162453)$ \\
\hline & & Aut $Q$ & $\{\varepsilon\}$ \\
\hline & & AAut $Q$ & $\varnothing$ \\
\hline & & $\langle I(Q)\rangle$ & $\begin{array}{l}(56),(3465),(3564),(356),(3645),(36)(45),(23),(23)(56), \\
(23)(465),(234),(2345),(23456),(235),(2364),(2365),(243), \\
(243)(56),(245),(246),(24)(35),(24)(36),(24536),(253)(46), \\
(254),(256),(2534),(25)(346),(25)(36),(2536),(2654),(264), \\
(264)(35),(26354),(2635),(26)(35)\end{array}$ \\
\hline
\end{tabular}




\begin{tabular}{|c|c|c|c|}
\hline № & $\mathrm{CL}$ & & Класс изотопии XVI \\
\hline \multirow[b]{4}{*}{1} & \multirow[b]{4}{*}{120} & $Q_{L}$ & $\varepsilon,(12)(3456),(132465),(1426)(35),(152364),(162543)$ \\
\hline & & Aut $Q$ & $\{\varepsilon\}$ \\
\hline & & AAut $Q$ & $(56)$ \\
\hline & & $\langle I(Q)\rangle$ & $\begin{array}{l}(56),(456),(465),(354),(35)(46),(364),(36)(45),(23)(56), \\
(23)(45),(23)(46),(234),(2345),(2346),(23564),(235)(46), \\
(23654),(236)(45),(243),(243)(56),(2453),(2463),(24)(56), \\
(253)(46),(256),(25634),(25346),(25364),(25436),(2536), \\
(263)(45),(265),(26534),(26345),(26354),(26435),(2635)\end{array}$ \\
\hline \multirow[b]{4}{*}{2} & \multirow[b]{4}{*}{120} & $Q_{L}$ & $\varepsilon,(12)(3456),(132465),(143526),(154)(236),(164253)$ \\
\hline & & Aut $Q$ & $\{\varepsilon\}$ \\
\hline & & AAut $Q$ & $(56)$ \\
\hline & & $\langle I(Q)\rangle$ & $\begin{array}{l}(56),(45),(456),(465),(46),(345),(3456),(3465),(346),(3564), \\
(356),(35)(46),(3654),(365),(36)(45),(23)(56),(2345),(2346), \\
(2354),(23564),(235),(23654),(2364),(236),(243)(56),(24563), \\
(24653),(245),(246),(2543),(253),(256),(2546),(254)(36), \\
(25)(36),(2536),(263),(265),(2645),(264)(35),(2635),(26)(35)\end{array}$ \\
\hline \multirow[b]{4}{*}{3} & \multirow[b]{4}{*}{120} & $Q_{L}$ & $\varepsilon,(12)(3456),(135246),(142653),(154)(236),(164325)$ \\
\hline & & Aut $Q$ & $\{\varepsilon\}$ \\
\hline & & AAut $Q$ & $\varnothing$ \\
\hline & & $\langle I(Q)\rangle$ & $\begin{array}{l}(456),(465),(34),(345),(3465),(3564),(356),(35)(46),(36), \\
(36)(45),(23),(234),(23564),(235),(235)(46),(2364),(2365), \\
(236),(236)(45),(243),(2463),(245),(24)(35),(24)(36),(253), \\
(25463),(254),(25),(256),(25)(46),(25)(346),(25436),(2536), \\
(26543),(2653),(26453),(264),(265),(265)(34),(2635),(26)(35)\end{array}$ \\
\hline \multirow[b]{4}{*}{4} & \multirow[b]{4}{*}{120} & $Q_{L}$ & $\varepsilon,(12)(3456),(136524),(1426)(35),(154623),(164325)$ \\
\hline & & Aut $Q$ & $\{\varepsilon\}$ \\
\hline & & AAut $Q$ & $\varnothing$ \\
\hline & & $\langle I(Q)\rangle$ & $\begin{array}{l}(456),(465),(34),(3456),(346),(35),(35)(46),(3654),(365), \\
(36)(45),(23),(234),(2354),(235),(2356),(235)(46),(23654), \\
(2365),(236),(236)(45),(243),(2453),(246),(24)(35),(24)(36), \\
(25643),(2563),(25463),(254),(256),(256)(34),(25)(36),(2536), \\
(263),(26453),(264),(265),(26),(26)(45),(26)(345),(26435), \\
(2635)\end{array}$ \\
\hline \multirow[b]{4}{*}{5} & \multirow[b]{4}{*}{12} & $Q_{L}$ & $\varepsilon,(123)(456),(135246),(143265),(1542)(36),(162534)$ \\
\hline & & Aut $Q$ & $\{\varepsilon\}$ \\
\hline & & AAut $Q$ & $\varnothing$ \\
\hline & & $\langle I(Q)\rangle$ & $\begin{array}{l}(45),(456),(465),(46),(354),(356),(364),(365),(36),(3645), \\
(36)(45),(234),(234)(56),(2345),(2453),(24),(24)(56),(246), \\
(24356),(246)(35),(24536),(253),(2564),(2546),(25)(34), \\
(256)(34),(254)(36),(25)(364),(25)(36),(2536),(2653),(263), \\
(26453),(265),(26),(2645),(26)(45),(2634),(26)(34),(26)(345), \\
(26)(354),(2635),(26)(35)\end{array}$ \\
\hline
\end{tabular}




\begin{tabular}{|c|c|c|c|}
\hline № & $\overline{\mathrm{CL}}$ & & Класс изотопии XVI \\
\hline \multirow[b]{4}{*}{6} & \multirow[b]{4}{*}{120} & $Q_{L}$ & $\varepsilon,(123)(456),(136524),(1462)(35),(154326),(163425)$ \\
\hline & & Aut $Q$ & $\{\varepsilon\}$ \\
\hline & & AAut $Q$ & $\varnothing$ \\
\hline & & $\langle I(Q)\rangle$ & $\begin{array}{l}(456),(346),(3564),(364),(36),(23),(23)(456),(2345),(23456), \\
(2346),(235),(2364),(2365),(23645),(236)(45),(243),(2456), \\
(246),(24)(356),(2435),(246)(35),(2436),(24536),(253)(46), \\
(25463),(2564),(2534),(25)(34),(25346),(2653),(263),(26453), \\
(263)(45),(2634),(26)(34),(26)(354),(26)(35)\end{array}$ \\
\hline \multirow[b]{4}{*}{7} & \multirow[b]{4}{*}{120} & $Q_{L}$ & $\varepsilon,(123)(456),(1365)(24),(146352),(154326),(162534)$ \\
\hline & & Aut $Q$ & $\{\varepsilon\}$ \\
\hline & & AAut $Q$ & $\varnothing$ \\
\hline & & $\langle I(Q)\rangle$ & $\begin{array}{l}(56),(45),(456),(34),(3456),(346),(354),(35),(364),(365), \\
(365),(36)(45),(23),(23)(56),(23)(45),(234)(56),(2354), \\
(235),(2356),(236),(236)(45),(2453),(2463),(24)(56),(245), \\
(2456),(24)(365),(24)(36),(253),(256),(2546),(2534),(25)(34), \\
(25)(346),(254)(36),(25436),(26543),(2643),(26453),(263)(45), \\
(2654),(264),(265),(26)(45),(26)(354)\end{array}$ \\
\hline \multirow[b]{4}{*}{8} & \multirow[b]{4}{*}{120} & $Q_{L}$ & $\varepsilon,(123)(456),(1365)(24),(143526),(153462),(163254)$ \\
\hline & & Aut $Q$ & $\{\varepsilon\}$ \\
\hline & & AAut $Q$ & (25) \\
\hline & & $\langle I(Q)\rangle$ & $\begin{array}{l}(456),(34)(56),(345),(3465),(354),(35),(35)(46),(364),(365), \\
(36),(3645),(23),(23)(45),(23)(456),(23)(46),(234),(23465), \\
(2346),(235),(236),(236)(45),(243),(2463),(2456),(24)(35), \\
(2435),(24356),(24)(365),(2543),(253),(256),(25)(46),(25)(34), \\
(25346),(26543),(2654),(264),(265),(26)(34),(264)(35)\end{array}$ \\
\hline \multirow[b]{4}{*}{9} & \multirow[b]{4}{*}{120} & $Q_{L}$ & $\varepsilon,(1234)(56),(135462),(145263),(1536)(24),(164325)$ \\
\hline & & Aut $Q$ & $\{\varepsilon\}$ \\
\hline & & AAut $Q$ & $\varnothing$ \\
\hline & & $\langle I(Q)\rangle$ & $\begin{array}{l}(465),(345),(354),(35),(3654),(23),(23)(465),(2345),(23465), \\
(2346),(2354),(2356),(235)(46),(23546),(236),(243),(245), \\
(2465),(2435),(24635),(24)(365),(2436),(245)(36),(253), \\
(2563),(253)(46),(25463),(254),(2534),(25)(34),(25)(364), \\
(25)(36),(26453),(263)(45),(2654),(2634),(26)(34),(26345)\end{array}$ \\
\hline
\end{tabular}

\begin{tabular}{|c|c|c|c|}
\hline № & $\mathrm{CL}$ & & Класс изотопии XVII \\
\hline \multirow{4}{*}{1} & \multirow{4}{*}{20} & $Q_{L}$ & $\varepsilon,(12)(3456),(135)(246),(14)(2365),(153)(264),(16)(2543)$ \\
\hline & & Aut $Q$ & $\{\varepsilon,(35)(46),(246),(24)(35),(264),(26)(35)\} \cong S_{3}$ \\
\hline & & AAut $Q$ & $\varnothing$ \\
\hline & & $\langle I(Q)\rangle$ & $(46),(35)(46),(24),(246),(24)(35),(264),(26),(26)(35)$ \\
\hline \multirow{4}{*}{2} & \multirow{4}{*}{60} & $Q_{L}$ & $\varepsilon,(12)(3456),(1365)(24),(146)(235),(1543)(26),(164)(253)$ \\
\hline & & Aut $Q$ & $\{\varepsilon,(35)(46)\} \cong Z_{2}$ \\
\hline & & AAut $Q$ & $\varnothing$ \\
\hline & & $\langle I(Q)\rangle$ & $(46),(35)(46),(23)(46),(235),(253),(25)(46)$ \\
\hline
\end{tabular}




\begin{tabular}{||c|c|c|l||}
\hline \hline № & CL & \multicolumn{1}{|c||}{ Класс изотопии XVII } \\
\hline \multirow{4}{*}{3} & \multirow{4}{*}{40} & $Q_{L}$ & $\varepsilon,(123)(456),(132)(465),(1425)(36),(1526)(34),(1624)(35)$ \\
\cline { 3 - 4 } & Aut $Q$ & $\{\varepsilon,(456),(465)\} \cong Z_{3}$ \\
\cline { 3 - 4 } & AAut $Q$ & $\varnothing$ \\
\cline { 2 - 4 } & & $\langle I(Q)\rangle$ & $(56),(45),(456),(465),(46),(23)(56),(23)(45),(23)(46)$ \\
\hline
\end{tabular}

\begin{tabular}{|c|c|c|c|}
\hline № & $\overline{C L}$ & & Класс изотопии XVIII \\
\hline \multirow[b]{4}{*}{1} & \multirow[b]{4}{*}{120} & $Q_{L}$ & $\varepsilon,(12)(3456),(135)(246),(14)(2365),(154326),(164253)$ \\
\hline & & Aut $Q$ & $\{\varepsilon\}$ \\
\hline & & AAut $Q$ & $\varnothing$ \\
\hline & & $\langle I(Q)\rangle$ & $\begin{array}{l}(56),(45),(456),(465),(46),(34),(34)(56),(3456),(35)(46), \\
(3654),(364),(23)(56),(234)(56),(23465),(2354),(235),(245), \\
(24635),(2436),(245)(36),(2543),(25643),(254),(25)(46), \\
(2534),(256)(34),(2536),(26543),(2654),(264),(265),(26534), \\
(26)(354),(26)(35)\end{array}$ \\
\hline \multirow[b]{4}{*}{2} & \multirow[b]{4}{*}{120} & $Q_{L}$ & $\varepsilon,(12)(3456),(135246),(142365),(1543)(26),(164)(253)$ \\
\hline & & Aut $Q$ & $\{\varepsilon\}$ \\
\hline & & AAut $Q$ & $\varnothing$ \\
\hline & & $\langle I(Q)\rangle$ & $\begin{array}{l}(56),(46),(34),(34)(56),(3456),(346),(35)(46),(3654),(364), \\
(36),(234),(234)(56),(2345),(23456),(235),(23546),(2463), \\
(245),(2465),(245)(36),(25643),(2563),(254),(2564),(25)(46), \\
(2534),(256)(34),(25)(346),(25346),(2654),(264),(265),(26), \\
(26534),(26)(34),(26)(35)\end{array}$ \\
\hline \multirow[b]{4}{*}{3} & \multirow[b]{4}{*}{60} & $Q_{L}$ & $\varepsilon,(12)(3456),(135246),(142365),(153264),(162543)$ \\
\hline & & Aut $Q$ & $\{\varepsilon,(35)(46)\} \cong Z_{2}$ \\
\hline & & AAut $Q$ & $\varnothing$ \\
\hline & & $\langle I(Q)\rangle$ & $\begin{array}{l}(56),(45),(456),(465),(46),(34),(3465),(346),(3564),(35)(46), \\
(364),(36),(234),(2346),(23564),(2356),(2463),(24),(246), \\
(24)(35),(24)(365),(2436),(2564),(256),(2534),(25346),(2654), \\
(264),(26),(2645),(26)(354),(26)(35)\end{array}$ \\
\hline \multirow[b]{4}{*}{4} & \multirow[b]{4}{*}{60} & $Q_{L}$ & $\varepsilon,(12)(3456),(136524),(146235),(154326),(164253)$ \\
\hline & & Aut $Q$ & $\{\varepsilon,(35)(46)\} \cong Z_{2}$ \\
\hline & & AAut $Q$ & $\varnothing$ \\
\hline & & $\langle I(Q)\rangle$ & $\begin{array}{l}\text { (56), (45), (456), (465), (46), (34), (3465), (346), (3564), (35)(46), } \\
(364),(36),(23)(456),(23)(46),(2345),(235),(2356),(2365), \\
(245),(2465),(2435),(24635),(2543),(253),(2563),(25)(46), \\
(2534),(25)(346),(2643),(2653),(263),(26453)\end{array}$ \\
\hline \multirow[b]{4}{*}{5} & \multirow[b]{4}{*}{120} & $Q_{L}$ & $\varepsilon,(12)(3456),(1365)(24),(143526),(154623),(164)(253)$ \\
\hline & & Aut $Q$ & $\{\varepsilon\}$ \\
\hline & & AAut $Q$ & $\varnothing$ \\
\hline & & $\langle I(Q)\rangle$ & $\begin{array}{l}(45),(456),(46),(345),(3465),(35)(46),(36),(36)(45),(23)(46), \\
(234),(2345),(2346),(2354),(235),(2365),(236),(243),(2453), \\
(24),(24)(56),(246),(246)(35),(24365),(24536),(253),(25346), \\
(25436),(2536),(2643),(2654),(2645),(26)(354)\end{array}$ \\
\hline
\end{tabular}




\begin{tabular}{|c|c|c|c|}
\hline № & $\mathrm{CL}$ & & К Класс изотопии XVIII \\
\hline \multirow{4}{*}{6} & \multirow{4}{*}{120} & $\overline{Q_{L}}$ & $\varepsilon,(123)(456),(134652),(1425)(36),(154326),(1624)(35)$ \\
\hline & & Aut $Q$ & $\{\varepsilon\}$ \\
\hline & & AAut $Q$ & $\varnothing$ \\
\hline & & $\langle I(Q)\rangle$ & $\begin{array}{l}(56),(45),(456),(465),(46),(3465),(3564),(35),(3546),(364), \\
(3645),(23)(56),(23)(45),(2345),(235)(46),(243),(24),(2465), \\
(246),(2543),(2564),(256),(25)(46),(2654),(264),(26),(2645)\end{array}$ \\
\hline \multirow[b]{4}{*}{7} & \multirow[b]{4}{*}{120} & $Q_{L}$ & $\varepsilon,(123)(456),(132465),(1436)(25),(1534)(26),(163542)$ \\
\hline & & Aut $Q$ & $\{\varepsilon\}$ \\
\hline & & AAut $Q$ & $\varnothing$ \\
\hline & & $\langle I(Q)\rangle$ & $\begin{array}{l}(56),(456),(465),(3456),(346),(354),(3546),(3654),(36),(3645), \\
(23)(56),(23)(46),(2356),(235)(46),(2453),(2435),(24635), \\
(254),(25),(2546),(25)(34),(25)(346),(25364),(25)(364), \\
(25)(36),(2536),(2653),(264),(2645),(26534),(26345)\end{array}$ \\
\hline \multirow[b]{4}{*}{8} & \multirow[b]{4}{*}{120} & $Q_{L}$ & $\varepsilon,(123)(456),(132465),(1426)(35),(154362),(1634)(25)$ \\
\hline & & Aut $Q$ & $\{\varepsilon\}$ \\
\hline & & AAut $Q$ & $\varnothing$ \\
\hline & & $\langle I(Q)\rangle$ & $\begin{array}{l}(456),(465),(34),(346),(3564),(35),(35)(46),(3546),(364), \\
(3645),(23),(23)(56),(23)(45),(234),(23564),(2356),(2463), \\
(2465),(24635),(246)(35),(24536),(2563),(253)(46),(25), \\
(2546),(25)(36),(2653),(264),(2645),(26345),(2635),(26)(35)\end{array}$ \\
\hline \multirow[b]{4}{*}{9} & \multirow[b]{4}{*}{120} & $Q_{L}$ & $\varepsilon,(1234)(56),(135246),(143625),(154263),(164532)$ \\
\hline & & Aut $Q$ & $\{\varepsilon\}$ \\
\hline & & AAut $Q$ & $\varnothing$ \\
\hline & & $\langle I(Q)\rangle$ & $\begin{array}{l}\text { (56), (46), (34), (34)(56), (345), }(3465),(3546),(3645),(36)(45), \\
(23),(23)(56),(234),(234)(56),(23456),(235),(2356),(236), \\
(243),(243)(56),(245),(2465),(246),(24)(36),(24365),(2543), \\
(254),(2564),(256),(2546),(25634),(26543),(2654),(264), \\
(2635)\end{array}$ \\
\hline \multirow[b]{4}{*}{10} & \multirow[b]{4}{*}{120} & $Q_{L}$ & $\varepsilon,(1234)(56),(136425),(146352),(154326),(162453)$ \\
\hline & & Aut $Q$ & $\{\varepsilon\}$ \\
\hline & & AAut $Q$ & $\varnothing$ \\
\hline & & $\langle I(Q)\rangle$ & $\begin{array}{l}(56),(34),(34)(56),(3456),(346),(356),(35)(46),(3546),(364), \\
(36),(3645),(23),(23)(56),(2345),(23456),(2346),(2354), \\
(23564),(235),(2365),(236),(2453),(24653),(2463),(245),(246), \\
(24)(35),(24635),(245)(36),(254),(2564),(254)(36),(2536), \\
(263),(2654),(264),(26354),(26435),(2635),(26)(35)\end{array}$ \\
\hline
\end{tabular}

\begin{tabular}{|c|c|c|c|}
\hline № & $\mathrm{CL}$ & & Класс изотопии XIX \\
\hline \multirow{4}{*}{1} & \multirow{4}{*}{60} & $Q_{L}$ & $\varepsilon,(12)(3456),(135)(246),(1436)(25),(153)(264),(1654)(23)$ \\
\hline & & Aut $Q$ & $\{\varepsilon,(35)(46)\} \cong Z_{2}$ \\
\hline & & AAut $Q$ & $=$ Aut $Q \cong Z_{2}$ \\
\hline & & $I(Q)$ & $\{\varepsilon,(35)(46),(246),(24)(35),(264),(26)(35)\} \cong S_{3}$ \\
\hline \multirow{4}{*}{2} & \multirow{4}{*}{20} & $Q_{L}$ & $\varepsilon,(12)(3456),(13)(2654),(146)(235),(15)(2436),(164)(253)$ \\
\hline & & Aut $Q$ & $\{\varepsilon,(35)(46),(23)(46),(235),(253),(25)(46)\} \cong S_{3}$ \\
\hline & & AAut $Q$ & $(46),(35),(23),(235)(46),(253)(46),(25)$ \\
\hline & & $I(Q)$ & $=$ Aut $Q \cong S_{3}$ \\
\hline
\end{tabular}




\begin{tabular}{||l|c|c|l||}
\hline \hline № & CL & \multicolumn{1}{|c||}{ Класс изотопии XIX } \\
\hline \multirow{3}{*}{3} & \multirow{4}{*}{40} & $Q_{L}$ & $\varepsilon,(123)(456),(132)(465),(1426)(35),(1524)(36),(1625)(34)$ \\
\cline { 3 - 4 } & Aut $Q$ & $\{\varepsilon,(456),(465)\} \cong Z_{3}$ \\
\cline { 3 - 4 } & & AAut $Q$ & $(56),(45),(46)$ \\
\cline { 3 - 4 } & & $I(Q)$ & $\{\varepsilon,(456),(465),(23)(56),(23)(45),(23)(46)\} \cong S_{3}$ \\
\hline
\end{tabular}

\begin{tabular}{|c|c|c|c|}
\hline № & $\mathrm{CL}$ & & Класс изотопии XX \\
\hline \multirow[b]{4}{*}{1} & \multirow[b]{4}{*}{60} & $Q_{L}$ & $\varepsilon,(12)(3456),(135246),(14)(2653),(15)(2364),(162543)$ \\
\hline & & Aut $Q$ & $\{\varepsilon,(25)(36)\} \cong Z_{2}$ \\
\hline & & AAut $Q$ & $\varnothing$ \\
\hline & & $\langle I(Q)\rangle$ & $\begin{array}{l}(45),(456),(34)(56),(3465),(356),(35)(46),(36),(36)(45), \\
(23)(465),(23)(46),(234),(23456),(23564),(235),(235)(46), \\
(23654),(2365),(236),(243)(56),(2453),(24563),(24),(2465), \\
(24)(35),(24)(365),(24)(36),(2543),(253),(2563),(25),(256), \\
(25)(46),(25)(34),(256)(34),(25)(36),(2536),(2643),(2653), \\
(263)(45),(2654),(265),(26)(45),(26)(34),(2635)\end{array}$ \\
\hline \multirow[b]{4}{*}{2} & \multirow[b]{4}{*}{120} & $Q_{L}$ & $\varepsilon,(12)(3456),(136524),(1435)(26),(1546)(23),(164253)$ \\
\hline & & Aut $Q$ & $\{\varepsilon\}$ \\
\hline & & AAut $Q$ & $\varnothing$ \\
\hline & & $\langle I(Q)\rangle$ & $\begin{array}{l}(45),(456),(46),(345),(3465),(346),(354),(35)(46),(36)(45), \\
(23)(45),(23)(456),(23)(46),(2346),(2354),(235),(2356), \\
(23654),(2364),(2365),(243),(24),(2465),(246),(24)(35),(2435), \\
(246)(35),(24)(36),(24536),(2543),(253),(2563),(253)(46), \\
(25),(25)(346),(25364),(25436),(2536),(2653),(2654),(2645), \\
(26345),(26)(354)\end{array}$ \\
\hline \multirow[b]{4}{*}{3} & \multirow[b]{4}{*}{30} & $Q_{L}$ & $\varepsilon,(12)(3456),(132546),(142653),(152364),(162435)$ \\
\hline & & Aut $Q$ & $\{\varepsilon,(3456),(35)(46),(3654)\} \cong Z_{4}$ \\
\hline & & AAut $Q$ & $\varnothing$ \\
\hline & & $\langle I(Q)\rangle$ & $\begin{array}{l}(456),(465),(345),(3456),(3465),(346),(354),(3564),(356), \\
(35)(46),(3546),(364),(365),(3645),(23)(46),(234),(234)(56), \\
(2345),(23456),(235),(2364),(2365),(23645),(24563),(245), \\
(2456),(246),(24)(35),(2435),(24356),(2436),(245)(36),(2543), \\
(253),(2563),(25463),(256),(25)(46),(2546),(25634),(256)(34), \\
(2653),(263),(263)(45),(2654),(264),(26534),(2634),(26345), \\
(26)(35)\end{array}$ \\
\hline \multirow[b]{4}{*}{4} & \multirow[b]{4}{*}{60} & $Q_{L}$ & $\varepsilon,(12)(3456),(132546),(1435)(26),(152364),(1653)(24)$ \\
\hline & & Aut $Q$ & $\{\varepsilon,(35)(46)\} \cong Z_{2}$ \\
\hline & & AAut $Q$ & $\varnothing$ \\
\hline & & $\langle I(Q)\rangle$ & $\begin{array}{l}(465),(46),(3456),(3465),(354),(3564),(35),(35)(46),(3546), \\
(3654),(364),(365),(3645),(23),(234),(2345),(2364),(2365), \\
(236),(2463),(245),(2456),(24)(35),(24)(36),(2436),(2543), \\
(2563),(254),(25),(256),(2546),(263),(2654),(2645),(26)(45), \\
(2634),(26)(35)\end{array}$ \\
\hline
\end{tabular}




\begin{tabular}{|c|c|c|c|}
\hline № & $\mathrm{CL}$ & & Класс изотопии $\mathbf{X X}$ \\
\hline \multirow[b]{4}{*}{5} & \multirow[b]{4}{*}{30} & $Q_{L}$ & $\varepsilon,(12)(3456),(136425),(143526),(154623),(165324)$ \\
\hline & & Aut $Q$ & $\{\varepsilon,(3456),(35)(46),(3654)\} \cong Z_{4}$ \\
\hline & & AAut $Q$ & $\varnothing$ \\
\hline & & $\langle I(Q)\rangle$ & $\begin{array}{l}(456),(465),(345),(3456),(3465),(346),(354),(3564),(356), \\
(35)(46),(3546),(364),(365),(3645),(23),(23)(45),(2345), \\
(2346),(2354),(235),(2356),(2365),(236),(243),(2453),(2463), \\
(24),(24)(56),(2456),(2465),(246),(2436),(2543),(253),(2563), \\
(254),(2564),(25),(2534),(25)(36),(2536),(2643),(2654),(264), \\
(265),(26),(2645),(2634),(26)(34),(2635)\end{array}$ \\
\hline \multirow[b]{4}{*}{6} & \multirow[b]{4}{*}{120} & $Q_{L}$ & $\varepsilon,(1234)(56),(1352)(46),(1425)(36),(154326),(162453)$ \\
\hline & & Aut $Q$ & $\{\varepsilon\}$ \\
\hline & & AAut $Q$ & $\varnothing$ \\
\hline & & $\langle I(Q)\rangle$ & $\begin{array}{l}(45),(46),(346),(35)(46),(3645),(23)(46),(23465),(2346), \\
(235),(235)(46),(23546),(23654),(2364),(2365),(2453),(24653), \\
(24)(56),(245),(2456),(24)(35),(2435),(2543),(253),(2563), \\
(256),(25)(46),(25364),(254)(36),(25436),(2536),(26543), \\
(2643),(2653),(26453),(2654),(264),(26),(2634),(26)(34), \\
(26354),(26435),(2635),(26)(35)\end{array}$ \\
\hline \multirow[b]{4}{*}{7} & \multirow[b]{4}{*}{120} & $Q_{L}$ & $\varepsilon,(1234)(56),(135462),(142536),(152643),(163245)$ \\
\hline & & Aut $Q$ & $\{\varepsilon\}$ \\
\hline & & AAut $Q$ & $\varnothing$ \\
\hline & & $\langle I(Q)\rangle$ & $\begin{array}{l}(45),(346),(354),(35),(35)(46),(36),(3645),(36)(45),(23)(46), \\
(234),(2346),(2354),(235),(2356),(23654),(2364),(236),(243), \\
(24)(56),(245),(24)(356),(2436),(245)(36),(2543),(253),(2563), \\
(253)(46),(25),(256),(25634),(25)(34),(25)(364),(25)(36),(26), \\
(2645),(26)(34),(26354),(26)(354),(2635)\end{array}$ \\
\hline
\end{tabular}

\begin{tabular}{|c|c|c|c|}
\hline № & $\overline{\mathrm{CL}}$ & \multicolumn{2}{|r|}{ Класс изотопии XXI } \\
\hline \multirow{4}{*}{1} & \multirow{4}{*}{6} & $Q_{L}$ & $\varepsilon,(1364)(25),(1435)(26),(1546)(23),(1653)(24)$ \\
\hline & & Aut $Q$ & $\begin{array}{l}\{\varepsilon,(3456),(35)(46),(3654),(23)(45),(23465),(2356),(2364), \\
(2463),(24)(56),(2435),(24536),(25643),(2546),(2534), \\
(25)(36),(2653),(2645),(26)(34),(26354)\} \cong Z_{5}\end{array}$ \\
\hline & & AAut $Q$ & $\varnothing$ \\
\hline & & $\langle I(Q)\rangle$ & $\begin{array}{l}(456),(465),(345),(3456),(3465),(346),(354),(3564),(356), \\
(35)(46),(3546),(364),(365),(3645),(23)(45),(234),(2345), \\
(2346),(2354),(235),(2364),(2365),(236),(243),(2453),(24)(56), \\
(245),(2456),(2465),(246),(2435),(2436),(2543),(253),(2563), \\
(254),(2564),(256),(2546),(25)(36),(2536),(2643),(2653),(263), \\
(2654),(264),(265),(2634),(26)(34),(2635)\end{array}$ \\
\hline
\end{tabular}




\begin{tabular}{||c|c|c|l||}
\hline \hline № & CL & \multicolumn{2}{|c||}{ Класс изотопии XXI } \\
\hline \multirow{5}{*}{2} & & $Q_{L}$ & $\varepsilon,(12)(3456),(13)(2546),(14)(2653),(15)(2364),(16)(2435)$ \\
\cline { 3 - 5 } & & Aut $Q$ & $\{\varepsilon,(3456),(35)(46),(3654)\} \cong Z_{4}$ \\
\cline { 2 - 4 } & AAut $Q$ & $\varnothing$ \\
\cline { 3 - 4 } & $\langle I(Q)\rangle$ & $(456),(465),(345),(3456),(3465),(346),(354),(3564),(356)$, \\
& & $(35)(46),(3546),(364),(365),(3645),(23)(56),(23)(46),(23456)$, \\
& & $(235),(23546),(23654),(2365),(24563),(24653),(246),(24)(35)$, \\
& & $(24)(36),(24365),(2436),(2543),(253),(25)(46),(25634)$, \\
& & $(25)(34),(25364),(25436),(26543),(2654),(264),(26)(45)$, \\
\hline
\end{tabular}

\begin{tabular}{||c|c|c|l||}
\hline \hline № & CL & \multicolumn{2}{|c||}{ Класс изотопии XXII } \\
\hline \multirow{4}{*}{1} & & $Q_{L}$ & $\varepsilon,(123)(456),(132)(465),(142635),(152436),(162534)$ \\
\cline { 3 - 4 } & \multirow{4}{*}{40} & Aut $Q$ & $\{\varepsilon,(456),(465)\} \cong Z_{3}$ \\
\cline { 3 - 4 } & AAut $Q$ & $(56),(45),(46)$ \\
\cline { 3 - 4 } & & $I(Q)$ & $\{\varepsilon,(456),(465),(23)(56),(23)(45),(23)(46)\} \cong S_{3}$ \\
\hline \hline
\end{tabular}

\section{Список литературы}

1. Albert A. A., Quasigroups II. Trans. Amer. Math. Soc. (1994) 55, 401-419.

2. Белоусов В. Д., Осповы теории квазигрупп и луп. Наука, Москва, 1967.

3. Coxeter H.S.M., Mozer W.O.J., Generators and relations for discrete groups. Springer, Berlin, 1972.

4. Dénes J., Keedwell A. D., Latin squares and their applications. Acad. Kiadö, Budapest, 1974.

5. Сафонова Л. В., Щукин К. К., Вычисление автоморфизмов и антиавтоморфизмов квазигрупп. Buletinul AS Rep. Moldova, Ser. Matematica (1990) 3(3), 49-55.

6. Schönhard E., Über Lateinische Quadrate und Unionen. J. Reine Angew. Math. (1930) 163, 183-229.

7. Sims C. C., Computational methods in the study of permutation groups. In: Comput. Probl. Abstract Algebra, Proc. Conf. Pergamon, Oxford, 1967, pp. 169-183.

Статья поступила 12.10.2000.

«ДИСКРЕТНАЯ МАТЕМАТИКА», 2002, ТОМ 14, ВЫПУСК 2

Заведующая редакцией Л. М. Барыкина

\begin{tabular}{|c|c|c|c|c|}
\hline Сдано в & 2002. & сано к печати 24.05.2 & Форм & $70 \times 100 / 16$ \\
\hline $\begin{array}{l}\text { Печать офсетная. } \\
\text { Тираж } 255 \text { экз. }\end{array}$ & Усл. печ. л. 13,0. & Усл. кр.-отт. 3,2 тыс. & Уч.-изд. л. 12,4. & $\begin{array}{r}\text { Бум. л. 5,0. } \\
\text { Заказ №6126. }\end{array}$ \\
\hline
\end{tabular}

Свидетельство о регистрации №1868 от 28.01.1991 г. в Госкомпечати СМ СССР.

Учредители: Академия наук СССР, Отделение математики

Адрес издательства: 117997 г. Москва, Профсоюзная ул., д. 90.

Адрес редакции: 117966 г. Москва ГСП-1, ул. Губкина, д. 8, комн. 622. Тел. 9383700.

Отпечатано в ППП "Тилография "Наука"

121099 г. Москва Г-99, Шубинский пер., д. 6. 